Bulletin of the Natural History Museum, 2018, 11: 21-61.

Received 25 Aug 2018; Accepted 22 Oct 2018.

doi:10.5937/bnhmb1811021S

UDC: $582.282 .16(497.11)$

Original scientific paper

\title{
CHECKLIST OF PEZIZOMYCETES FROM SERBIA
}

\author{
DRAGIŠA SAVIĆ ${ }^{*}$, ISKRA KAJEVSKA ${ }^{2}$, NENAD MILOSAVLJEVIĆ ${ }^{3}$ \\ ${ }^{1}$ National Park Fruška Gora, Zmajev trg 1, 21208 Sremska Kamenica, Republic of \\ Serbia, e-mail: dragita@gmail.com \\ ${ }^{2}$ University of Niš, Faculty of Sciences and Mathematics, Department of Biology \\ and Ecology, Višegradska 33, 18000 Niš, Republic of Serbia, e-mail: \\ iskra.kajevska@pmf.edu.rs \\ ${ }^{3}$ Ivana Milutinovića 23, 34000 Kragujevac, Republic of Serbia, e-mail: \\ nenadivesna@yahoo.com
}

In this paper, report on species distribution from the class Pezizomycetes is presented, in the Republic of Serbia. The list of species contains all known published and unpublished data so far. To date, a total number of 206 species have been listed, of which even 105 have been recognized as new country records. This comprehensive species list significantly enriches the knowledge of this taxonomic group in the country.

Key words: Fungi, Ascomycota, Southeast Europe.

\section{INTRODUCTION}

The fundamental classification within Ascomycota was based upon the structure of the ascus apical apparatus and the mode of dehiscence of the ascus. These features were a significant taxonomic character in the 
delimitation of Pezizomycetes, commonly known as operculate discomycetes, from the inoperculate discomycetes or currently known as Leotiomycetes (Landvik et al. 1997). Classifications within Pezizomycetes have long been based exclusively on morphology and cytology (e.g. Boudier 1907, Nannfeldt 1932, Eckblad 1968, Spatafora et al. 2006). Today, after numerous molecular phylogenetic studies, it has been accepted that this ascomycetous class is an early diverging lineage within the largest subphylum Pezizomycotina in the kingdom of fungi (Spatafora et al. 2006, Hansen \& Pfister 2006, Hansen et al. 2013) with a single order Pezizales containing 16 currently accepted families (Kirk et al. 2008, Pfister 2015). They are omnipresent in nature occurring in a broad range of habitats with epigeous, semihypogeous and hypogeous ascoma fruiting on soil, wood and leaf debris, dung, herbaceous plants, trees, bryophytes, lichens. Hence, taxa of this ascomycetous class are with diverse trophic strategies, ranging from saprotrophic, mycorrhyzal, bryoparasitic, phytoparasitic (Hansen \& Pfister 2006, Tedersoo 2013).

The southeastern European country Serbia is situated in the central part of the Balkan Peninsula consisting of highlands and mountains as well as in the southern part of the Pannonian Plain prevailing by broad fertile plains. This specific geographic position, characterized with complex geology and relief as well as remarkable diversity and divergent ecological habitats dominated by different climatic influences, undoubtedly contributes to a particularly rich mycological diversity in the country. The territory of Serbia regarding mycodiversity is still insufficiently explored. Checklist on this fungal class does not exist as Pezizomycetes are frequently overlooked hence the knowledge on taxa of this fungal group in the country is poorly known. Therefore, the aim of this study is to compile an annotated checklist of the recently registered species as well as unpublished data of Pezizomycetes in Serbia.

The first data on fungi of the class Pezizomycetes from Serbia were published by Schulzer et al. (1866). In this work, 8 pezizalean species are listed but only 4 of them have been included in our list because the latin names of the rest of them (Ascobolus vernalis, Helvella cionereocandida, Peziza bichroa and Otidea dehiscens) cannot be linked to today's accepted names/synonyms of fungi. Two remarkable works on mycodiversity with special emphasis on fungal plant pathogens mainly from Ascomycota have been published by Ranojević $(1910,1914)$ but with scarce data (only eight species) on Pezizomycetes. Afterward, until the end of the $20^{\text {th }}$ century, only a few works have been published with scattered data on Pezizomycetes (e.g. Čolić 1967, Gajić 1984, 1989, Beronja 1995, Matavulj 1995, Matavulj et al. 1995). During the decade of the 2000s, the number of mycological studies has been increased. Specifically, important data on 
some Pezizomycetes, mainly fungal pathogens on forest trees, have been summarized by Karadžić (2003), Karadžić \& Milijašević (2004), Karadžić $\&$ Stanivuković (2010). Subsequently, papers concerning valuable information are as follows: Ławrynowicz et al. (1997), Benkert (2006, 2007), Milenković et al. (2015), Milosavljević (2016, 2017).

Recently, major comprehensive research of Ascomycota in Serbia has been conducted in NP Fruška Gora, situated in the northern part of Serbia, with published data on 87 taxa of the class Pezizomycetes in Karaman et al. (2012) and Savić (2016). These publications have substantially enriched the knowledge of the presence of these fungi in the country. Data from the doctoral thesis Savić (2016), who is one of the authors of this paper, are published in this paper, and therefore, those species are not specifically indicated but are included together with other new findings.

Additionally, noteworthy are data of two new recently described species on the basis of samples collected in Serbia. These are Tuber petrophilum (Milenković et al. 2015) and Octospora pannosa (Vega et al. 2018).

\section{MATERIALS AND METHODS}

The species list has been composed based on all known country's published data on Pezizomycetes and unpublished data from the author's own findings on the area of the Republic of Serbia. In addition, many amateur mycologists contributed to this study by sending samples collected from various parts of Serbia on identification to the authors of this paper.

All specimens were analyzed in a fresh state according to the standard methods used in fungal identification. Spores and other elements have been examined and measured in water, $5 \% \mathrm{KOH}$, Lugol's solution, Melzer's and Lactophenol Cotton Blue reagents. These analyses were obtained in a bright field microscopy with the use of BIM313T - LED and Optika N400M. After identification; most of the specimens were dried and deposited in several fungariums. The acronym F.G. refers to the unregistered fungarium of the National park Fruška Gora, the acronym N.M. are from the private fungarium of Nenad Milosavljević and the acronym M.S.N. represents the unregistered fungarium of the Mycological Society of Niš.

Identification was confirmed by using the works of Dennis (1981), Breitenbach \& Kränzlin (1981), Jacobson et al. (1998), Hansen \& Knudsen (2000), Montecchi \& Sarasini (2000), Doveri (2004), Schumacher (1990) and Olariaga et al. (2015). 
Families, genera, and species are listed in alphabetical order. Each individual specimen in the species list is represented primarily with the name of the wider area (eg. Fruška Gora Mt.) followed by the name of a one or more specific sites displayed in brackets (the abbreviation vill. indicates a name of a village and $\mathrm{r}$. - a river). Subsequently, the collection date, substrate, the number of fungal specimen and the names of the collector (Leg.) and the determinator (Det.) are indicated. References, page number, and other relevant data are included for previously reported species. Species which represent first record on the territory of Serbia are marked with an asterisk $\left(^{*}\right)$ in front of the name.

Names of authors of fungal taxa are abbreviated according to Kirk \& Ansell (2004), including its online version derived from the Index Fungorum website.

\section{RESULTS AND DISCUSSION}

From 16 known and accepted families within the single order Pezizales we have listed species from 12 families. These species are at present distributed in 62 genera. The family Pyronemataceae is the most genera rich with a total number of 28 genera and it is a family with the highest number of species represented remarkably with 78 taxa. Other families are Pezizaceae with 7 genera and 31 species, followed by Ascobolaceae with 3 genera and 22 species, Helvellaceae - 2 genera and 21 species and Tuberaceae with 3 genera and a total number of 19 species. Related to Peziza species number, the Peziza varia-cerea-repanda-micropus complex has always been inconvenient to distinguish. According to Hansen et al. (2002), ITS and morphological analyses confirmed that the name $P$. repanda should be rejected and the rest of the taxa should not be considered as separate but as one variable species $P$. varia. Although DNA evidence is very convincing (Beug et al. 2014) this concept has not yet been widely accepted probably due to a lack of more comprehensive analyzes in this aspect. Therefore, we listed these Peziza species separately until their unification will be well supported and solved.

Species data has been obtained from different parts of the country, but the vast majority originates from the mountain Fruška Gora in the northern part of the country, mountains Goč and Rudnik (central Serbia) and mountains around the city of Niš (southeast Serbia). In addition, family Pyronemataceae is represented with most new records for the country, total of 50 species, followed by Ascobolace (21) and Pezizaceae with 18 species as new for the country. 
Due to insufficient data, it is not possible to analyze and estimate the prevalence and the number of certain species in Serbia at this moment. For the same reason, only 6 species from the class Pezizomycetes are currently on the list of a protected species of fungi in Serbia (Ivančević et al. 2012). Considering that in this paper all existing data are summarized, this work can be considered as a starting point for all future research of these fungi in Serbia. It will also contribute into a better understanding of the recent status as well as during future revision of the list of protected species of fungi in Serbia.

\section{Species List}

Class Pezizomycetes O.E. Erikss. \& Winka 1997

Ordo: PeZIZALES J. Schröt. 1897

Fam.: Ascobolaceae Boud. ex Sacc. 1884

1.*Ascobolus albidus P. Crouan \& H. Crouan

Fruška Gora Mt. (Brankovac), 17.03.2015., on horse dung, F.G. 100 , leg. \& det. D. Savić.

2.*Ascobolus brassicae P. Crouan \& H. Crouan

Fruška Gora Mt. (Ledinci vill.), 08.11.2017., on mouse dung, F.G. 715, leg. \& det. D. Savić.

3.*Ascobolus carbonarius P.Karst.

Fruška Gora Mt. (Testera), 18.09.2014., on Quercus petraea, burned wood, F.G. 208, leg. \& det. D. Savić; Fruška Gora Mt. (Stražilovo), 02.07.2015., on deciduous tree, F.G. 291, leg. \& det. D. Savić.

4.*Ascobolus crenulatus P.Karst.

Fruška Gora Mt. (Andrevlje), 29.01.2013., on Capreolus capreolus dung, leg. \& det. D. Savić.

5. Ascobolus foliicola Berk. \& Broome

Beograd (Topčider), sub Ascobolus serbicus, on Rubus sp, Ranojević, 1902: 103.

6.*Ascobolus furfuraceus Pers.

Fruška Gora Mt. (Brankovac), 17.03.2015., on horse dung, F.G. 189, leg. \& det. D. Savić; Niš (Cerje vill.), 21.11.2015., on cow dung, M.S.N. 113, leg. \& det. I. Kajevska; Fruška Gora Mt. (Bešenovački prnjavor vill.), 25.11.2016., on horse dung, F.G. 508, leg. \& det. D. Savić; Kopaonik Mt. (Gobelja), 01.09.2018., on cow dung, F.G: 837, leg. \& det. D. Savić. 
6.*Ascobolus hansenii M.D. Paulsen \& Dissing Fruška Gora Mt. (Beočin monastery), 15.06.2018., on soil, leg. \& det. D. Savić.

8.*Ascobolus immersus Pers.

Fruška Gora Mt. (Paragovo), 25.12.2012., on horse dung, leg. \& det. D. Savić; Fruška Gora Mt. (Glavica), 19.03.2018., on sheep dung, leg. \& det. D. Savić.

9.*Ascobolus lignatilis Alb. \& Schwein.

Fruška Gora Mt. (Stražilovo), 02.11.2015., on Populus alba, twig, F.G. 321, leg. \& det. D. Savić.

10.*Ascobolus michaudii Boud.

Kopaonik Mt. (Gobelja), 01.09.2018., on cow dung, F.G. 857, leg. $\&$ det. D. Savić.

11.*Ascobolus perplexans Massee \& E.S. Salmon

Zasavica, 20.06.2017., on soil, F.G. 686, leg. \& det. D. Savić; Fruška Gora Mt. (Testera), 18.10.2017., on soil, F.G. 724, leg. \& det. D. Savić.

12.*Ascobolus roseopurpurascens $\mathrm{Rehm}$

Fruška Gora Mt. (Brankovac), 17.03.2015., on horse dung, F.G. 109, leg. \& det. D. Savić.

13.*Ascobolus sacchariferus Brumm.

Fruška Gora Mt. (Ravne), 04.04.2013., on Cervus elaphus dung, leg. \& det. D. Savić; Fruška Gora Mt. (Paragovo), 19.03.2015., on Lepus europaeus dung, F.G. 89, leg. \& det. D. Savić.

14.*Ascobolus viridis Curr.

Fruška Gora Mt. (Papratski do), 08.05.2018., on wet ground, leg. $\&$ det. D. Savić

15.*Saccobolus citrinus Boud. \& Torrend

Fruška Gora Mt. (Bukovac vill.), 19.04.2018., on sheep dung, leg. $\&$ det. D. Savić.

16.*Saccobolus depauperatus (Berk. \& Broome) E.C. Hansen Fruška Gora Mt. (Vorovo), 04.04.2018., on Cervus elaphus, dung, F.G. 894, leg. \& det. D. Savić; Kopaonik Mt. (Gobelja), 01.09.2018., on cow dung, leg. \& det. D. Savić.

17.*Saccobolus dilutellus (Fuckel) Sacc.

Fruška Gora Mt. (Crveni čot), 26.02.2017., on Lepus europaeus, dung, F.G. 800, leg. \& det. D. Savić. 
18. ${ }^{*}$ Saccobolus minimus Vel.

Fruška Gora Mt. (Iriški venac), 10.12.2018., Capreolus capreolus, dung, leg. \& det. D. Savić.

19. *Saccobolus versicolor (P.Karst.) P.Karst.

Fruška Gora Mt. (Bukovac vill.), 18.04.2018., on sheep dung, F.G. 799, leg. \& det. D. Savić.

20.*Thecotheus crustaceus (Starbäck) Aas \& N. Lundq.

Fruška Gora Mt. (Brankovac), 10.03.2014., on horse dung, leg. \& det. D. Savić.

21.*Thecotheus pelletieri (P.Crouan \& H.Crouan) Boud.

Fruška Gora Mt. (Paragovo), 11.02.2013., on horse dung, leg. \& det. D. Savić; Fruška Gora Mt. (Kraljeva stolica), 26.09.2014., on horse dung, F.G. 73, leg. \& det. D. Savić.

22.*Thecotheus rivicola (Vacek) Kimbr. \& Pfister

Fruška Gora Mt. (Stražilovo), 23.08.2013., on soil, leg. \& det. D. Savić.

Fam.: Ascodesmidaceae J. Schröt. 1893

23. ${ }^{*}$ Lasiobolus papillatus (Pers.) Sacc.

Fruška Gora Mt. (Brankovac), 16.03.2015., on horse dung, F.G. 55, leg. \& det. D. Savić; Suva Planina Mt. (Bojanine Vode), 22.08.2017., on cow dung, M.S.N. 204, leg. \& det. I. Kajevska.

24. ${ }^{*}$ Pseudoboubovia benkertii (B. Perić) U. Lindem., M. Vega, B. Perić \& Tena

Zasavica, 16.03.2015., on soil between the reed, F.G. 685, leg. \& det. D. Savić.

Fam.: Caloscyphaceae Harmaja 2002

25. ${ }^{*}$ Caloscypha fulgens (Pers.) Boud.

Goč Mt. (Brezjak), 22.05.2015., leg. \& det. N. Milosavljević; Goč Mt. (Brezna vill.), 15.04.2018, N.M. 20180415-1, leg. V. Milosavljević, det. N. Milosavljević.

Fam.: Discinaceae Benedix 1962

26.*Discina ancilis (Pers.) Sacc.

Fruška Gora Mt. (Iriški venac), 11.04.2013., on Pinus nigra, wood, leg. \& det. D. Savić; Fruška Gora Mt. (Paragovo), 16.10.2003., leg. \& det. D. Savić; Deliblatska peščara (Crni vrh), 10.04.2013., leg. \& det. D. Savić; Goč Mt. (Stojanac), 04.04.2015, 
leg. \& det. N. Milosavljević; Fruška Gora Mt. (Vizić vill.), 20.03.2018., F.G. 850, leg. J. Gajić, det. D. Savić.

27. Discina fastigiata (Krombh.) Svrček \& J.Moravec Beograd (Zvezdara), 27.04.2004., leg. \& det. I.Hadžić; Fruška Gora Mt. (Letenka), sub Gyromitra infula (incorrect determination), Karaman et al. 2012: 42; Deliblatska peščara (Crni vrh), 10.04.2013., leg. \& det. D. Savić; Fruška Gora Mt. (Vizić vill.), 20.03.2018., F.G. 807, leg. J. Gajić, det. D. Savić.

28. Gyromitra esculenta (Pers.) Fr.

Tara Mt. (Crveni potok), Čolić 1967: 411; Goč Mt. (Golići), 05.04.2014, leg. \& det. N. Milosavljević; Goč Mt., Gajić 1984: 45; Babušnica (Stol vill.), 04.2015., on soil, leg. \& det. D. Stojanović; Fruška Gora Mt. (Paragovo), Karaman et al. 2012: 42; Kopaonik Mt. (Jankove bare), 30.08.2018., F.G. 885, leg. S. Baluban, det. D. Savić.

29. Gyromitra gigas (Krombh.) Cooke

Tara Mt. (Crveni potok), Čolić 1967: 411; Beograd (Lipovička šuma), 06.05.2002., leg. \& det. I. Hadžić; Rudnik Mt. (Bare vill.), 05.04.2013, leg. \& det. N. Milosavljević; Goč Mt. (Cvetne livade), 24.04.2014., leg. V. Milosavljević, det. N. Milosavljević; Niš (Gradac), 04.2015., on soil, leg. \& det. M. Kuštera.

30. Gyromitra infula (Schaeff.) Quél.

Tara Mt. (Kaluđerske bare), Ranojević 1910: 352; Tara Mt. (Crveni potok), sub Helvella infula, Čolić 1967: 411.

31. Gyromitra parma (J.Breitenb.\& Maas Geest) Kotl. \& Pouzar Beograd (Bojčinska šuma), sub Discina parma, Ivančević \& Davidović 2011: 24; Fruška Gora Mt. (Popovica), 15.04.2013., on deciduous tree, rotten wood, leg. \& det. D. Savić; Suva planina Mt. (Ploče), 04.2015., on soil, leg. R. Lukić, det. M. Kuštera; Niš (Brzi Brod), along Nišava r., 04.2018., on soil, leg. \& det. S. Lazarević.

32.* Gyromitra ticiniana Littini

Rudnik Mt. (Lipovac vill.), 20.04.2013., leg. \& det. N. Milosavljević; Niš (Gabrovac vill.), 30.03.2017., leg. S. Cekić, det. I. Kajevska; Niš (Čamurlija vill.), 04.2015., on soil, M.S.N. 172, leg. S. Lazarević, det. M. Kuštera.

33. Hydnotrya cerebriformis Harkn.

Tara Mt., Ivančević 2016: 82. 
Fam.: Helvellaceae Fr. 1822

34. Balsamia vulgaris Vittad.

Obrež vill., Lajkovac (Markova crkva vill.) and Lipik-Umka (Ivančević 2016: 70).

35. Helvella acetabulum (L.) Quelet

Beograd (Ada Ciganlija), 22.04.1989., leg. \& det. I. Hadžić; Fruška Gora Mt. (Bukovac vill. and Glavica), Karaman et al. 2012: 42; Fruška Gora Mt. (Iriški venac), 23.05.2014., F.G.67, leg. \& det. D. Savić; Niš (Gabrovac vill.), 09.05.2017., on soil, M.S.N. 178, leg. N. Pavković, det. I. Kajevska; Beograd (Botanical Garden), Vukojević et al. 2016: 252; Kragujevac (Žeželj), Milosavljević 2016: 82.

36. Helvella atra J. König

Fruška Gora Mt. (Sremska Kamenica), Schulzer et al. 1866: 63; Beograd (Moštanica), 13.11.1999., leg. \& det. I. Hadžić; Fruška Gora Mt. (Rakovac vill.), 29.11.2013., leg. \& det. D. Savić; Suva planina (Ploča), 06.2015., on soil, M.S.N. 225, leg. I. Cvijetan, det. M. Kuštera; Niš (Prosek vill.), 15.07.2018., on soil, M.S.N. 232, leg \& det. I. Kajevska; Goč Mt. (Cvetne livade), Milosavljević 2016: 60; Jelova Gora Mt. (Gostinica vill.), 13.07.2018., F.G. 815, leg. M. Adžić, det. D. Savić.

37. Helvella bicolor Raddi

Fruška Gora Mt. (Sremska Kamenica), sub H. elastica var. fusca (probably incorrect determination), Schulzer et al. 1866: 63; Fruška Gora Mt. (Paragovo), sub H. elastica (incorrect determination), Karaman et al. 2012: 42; Fruška Gora Mt. (Andrevlje), 31. 05.2013., leg. \& det. D. Savić; Niš (Gabrovac vill.), 09.05.2017., on soil, M.S.N. 179, leg. \& det. I. Kajevska.

38. Helvella costifera Nannf.

Kragujevac (Žeželj), Milosavljević 2016: 83; Niš (Čamurlija vill.), 17.04.2018., on soil, leg. S. Lazarević, det. Iskra Kajevska.

39. Helvella crispa (Scop) Fr.

Tara Mt. (Crveni potok), Čolić 1967: 411; Fruška Gora Mt. (Glavica, Ledinci, Paragovo and Sremska Kamenica), Karaman et al. 2012: 42; Avala Mt. (Zuce), 28.09.2014., leg. \& det. I. Hadžić; Niš (Cerje vill.), 17.10.2015., leg. \& det. I. Kajevska; Gledićke planine Mt. (Adžine livade vill.), Milosavljević 2016: 70.

40. Helvella elastica Bull.

Tara Mt. (Crveni potok), sub Leptopodia elastica, Čolić 1967: 413; Kopaonik Mt. (Gobelja), 11.09.2015., leg. \& det. I. Kajev- 
ska; Niš (Cerje vill.), 17.10.2015., on soil, M.S.N. 221, leg. \& det. I. Kajevska; Niš (Malča vill. along Nišava r.), 27.07.2018., on soil, M.S.N. 243, leg \& det. I. Kajevska; Brus, 03.09.2016., leg. \& det. M. Slavova; Goč Mt. (Dobre vode), Milosavljević 2016: 63.

\section{Helvella fibrosa (Wallr.) Korf}

Fruška Gora Mt. (Sviloš vill.), 22.04.2013., leg. \& det. D. Savić; Fruška Gora Mt. (Stražilovo), 22.05.2014., F.G. 69, leg. \& det. D. Savić; Goč Mt. (Brezna vill. and Cvetne livade), Milosavljević 2016: 58; Brus (Tršanovci vill.), 19.06.2016., on soil, M.S.N. 163, leg. \& det. I. Kajevska.

\section{Helvella fusca Gillet}

Niš (Brzi brod), 04.2015., on soil, leg. \& det. M. Kuštera; Kragujevac (Žeželj), Milosavljević 2016: 80.

43. Helvella hypocrateriformis Schaeff.

Goč Mt. (Brezjak and Prerovo), sub Helvella cupuliformis, Milosavljević 2016: 55; Fruška Gora Mt. (Paragovo), 03.07.2018., leg. \& det. D. Savić.

44. Helvella lactea Boud.

Rudnik Mt. (Bare vill.), Milosavljević 20115: 78 and Milosavljević 2016: 75.

\section{Helvella lacunosa Afzelius}

Stara planina Mt. (Dojčinovo vrelo-Jabučko ravnište), Ivančević \& Beronja 2007: 16; Fruška Gora Mt. (Glavica, Sremska Kamenica and Vrdnik vill.), Karaman et al. 2012: 42; Niš (Kamenički vis), 15.10.2016., on soil, M.S.N. 126, leg. M. Kuštera, det. I. Kajevska; Stara planina Mt. (Ponor), 25.06.2016., on soil, leg. N. Pavković, det. I. Kajevska; Niš (Prosek vill.), on soil, 15.07.2018., M.S.N. 233,234, leg. \& det. I. Kajevska; Goč Mt. (Prerovo and Brezjak), Milosavljević 2016: 74; Gledićke planine Mt. (Adžine livade vill.), Milosavljević 2016: 74; Kukavica Mt. (Slatina vill.), 04.06.2017., on soil, leg. \& det. S. Jovanović; Vlasotince (Brezovica vill.), 28.05.2018., leg. \& det. A. Peškić; Vlasinsko jezero (Vlasina okruglica vill.), 05.08.2018., leg. \& det. A. Peškić; Dimitrovgrad (Praca vill.), 26.07.2018., leg. \& det. M. Petrov; Jelova Gora Mt. (Gostinica vill.), 01.08.2018., leg. M. Adžić, det. D. Savić. 
46.*Helvella leucomelaena (Pers.) Nannf.

Fruška Gora Mt. (Bukovac vill.), 05.04.2009., leg. \& det. D. Savić; Deliblatska peščara, 23.03.2014., leg. \& det. I. Hadžić; Babušnica (Vava vill.), 04.2015., on soil, leg. \& det. D. Stojanović; Niš (Kamenički vis), 14.05.2016., on soil, M.S.N. 207, leg. S. Lazarević, det. I. Kajevska; Fruška Gora Mt. (Ledinci vill.), 09.04.2018., F.G. 836, leg. \& det. D. Savić; Fruška Gora Mt. (Letenka), 12.04.2018., F.G. 806, leg. \& det. D. Savić.

47.*Helvella levis Bergeret

Fruška Gora Mt. (Papratski do), 03.06.2012., leg. \& det. D. Savić; Fruška Gora Mt. (Andrevlje), 03.06.2013., F.G. 56, leg. \& det. D. Savić; Šabac (Mali Zabran), 19.05.2016., on soil, leg. \& det. S. Stanojević; Beograd (Bojčinska šuma), 24.10.2015., leg. \& det. I. Hadžić; Suva planina Mt. (Gadžin han), 29.05.2016., on soil, M.S.N. 139, leg. \& det. I. Kajevska; Niš (Prosek vill.), on soil, 15.07.2018., M.S.N. 235, leg. \& det. I. Kajevska; Suva planina Mt. (Bojanine Vode), on soil, 03.08.2018., M.S.N. 247, leg. \& det. I. Kajevska; Fruška Gora Mt. (Ravne), 07.06.2018., F.G. 844, leg. \& det. D. Savić; Fruška Gora Mt. (Paragovo), 05.07.2018., leg. \& det. D. Savić.

48. Helvella macropus (Pers.) P. Karst.

Beograd (Velika Moštanica), 13.11.1999., leg. \& det. I. Hadžić; Goč Mt. (Brezjak and Dobre vode), Milosavljević 2016: 91; Krupanj, 23.07.2018., leg. M. Marković, det. D. Savić.

49. Helvella monachella (Scop.) Fr.

Subotičko-horgoška peščara (Bukvać), Gajić 1986: 28; Deliblatska peščara, 27.04.2004., leg. \& det. I. Hadžić; Fruška Gora Mt. (Paragovo), Karaman et al. 2012: 42; Niš (Brzi brod), 04.2015., leg. \& det. det. M. Kuštera; Rudnik Mt. (Vraćevšnica monastery), Milosavljević 2016: 66; Fruška Gora Mt. (Susek vill.), 20.04. 2018., F.G. 808, leg. \& det. D. Savić; Fruška Gora Mt. (Beočin), 24.04.2018., leg. \& det. D. Savić.

50. Helvella oblongispora Harmaja

Goč Mt. (Cvetne livade), Milosavljević 2016: 87.

51.*Helvella pezizoides Afzel.

Fruška Gora Mt. (Stražilovo), 03.70.2013., leg. \& det. D. Savić.

52. Helvella solitaria P. Karst

Beograd (Bojčinska šuma), Ivančević \& Davidović 2011: 25; Suva planina (Ploča), 05.2015., leg. N. Pavković, det. M. Kuštera; 
Kragujevac (Žeželj), Milosavljević 2016: 88; Niš (Knez vill.), 17.05.2017., on soil, M.S.N. 186, leg. \& det. I. Kajevska; Kukavica Mt. (Slatina vill.), 09.05.2017., on soil, leg. \& det. S. Jovanović; Fruška Gora Mt. (Rakovac vill.), 24.04.2018., F.G. 830, leg. \& det. D. Savić.

53. Helvella sublicia Holmsk.

Goč Mt. (Cvetne livade and Brezjak), sub H. ephippium, Milosavljević 2016: 64; Niš (Gabrovac vill.), 04.06.2017., on soil, M.S.N. 198, leg. \& det. I. Kajevska.

54. Helvella sulcata Afzel.

Kragujevac (Žeželj), Milosavljević 2016: 76.

Fam.: Morchellaceae Rchb. 1834

55. Disciotis venosa (Pers.) Arnould

Knić (Gruža Lake), 13.04.2008., leg. G. Bogićević, det. N. Milosavljević \& N. Lukić; Beograd (Bojčinska šuma), Ivančević \& Davidović 2011: 24; Fruška Gora Mt. (Iriški venac, Sremska Kamenica and Beočin), Karaman et al. 2012: 42; Goč Mt. (Prerovo), 13.04.2017., leg. \& det. N. Milosavljević; Fruška Gora Mt. (Brazilija vill.), 12.04.2018., F.G. 801, leg. \& det. D. Savić; Niš (Brzi Brod), along Nišava r., on soil, 13.04.2018., M.S.N. 223, leg. \& det. S. Lazarević.

56. Fischerula macrospora Mattir.

Tara Mt. (Mitrovac), Ivančević 2016: 78.

57.* Morchella deliciosa $\mathrm{Fr}$.

Beograd (Ada ciganlija), 30.03.1994., leg. \& det. I. Hadžić; Kragujevac (Šumarice), 03.04.2009., leg. \& det. N. Milosavljević; Goč Mt. (Strugara), 10.04.2014., leg. \& det. N. Milosavljević.

58. Morchella elata Fr.

Tara Mt. (Crveni potok), Čolić 1967: 413; Beograd (Ada ciganlija), 21.04.1993., leg. \& det. I. Hadžić; Fruška Gora Mt. (Sremska Kamenica), sub Morchella costata, Karaman et al. 2012: 42; Goč Mt. (Jezero), 15.04 2014., leg. \& det. N. Milosavljević; Fruška Gora Mt. (Beočin), 06.04.2017., leg. \& det. D. Savić.

59. Morchella esculenta (L.) Pers.

Goč Mt., Gajić 1984: 45; Golija Mt., Gajić 1989: 81; Fruška Gora Mt., Ranković 1955; Klještevica vill., 25.04.1997., leg. \& det. I. Hadžić; Beograd (Jajinci), 27.04.1998., leg. \& det. I. Hadžić; Beograd (Titov gaj), 07.04.2003., leg. \& det. I. Hadžić; Knić 
(Gruža Lake), 13.04.2008. leg. G. Bogićević, det. N. Milosavljević \& N. Lukić; Fruška Gora Mt. (Grgurevci vill.), 25.03.2010., leg. \& det. D. Savić; Beograd (Bojčinska šuma), Ivančević \& Davidović 2011: 25; Fruška Gora Mt. (Bukovac vill.), sub M. crassipes, Karaman et al. 2012: 42; Klenak (Tursko groblje), 22.04.2012., leg. \& det. S. Stanojević; Petrovaradinski rit, sub $M$. vulgaris, Karaman et al. 2012: 42; Rudnik Mt. (Dobrača vill.), 17.04.2013,. leg. \& det. N. Milosavljević; Goč Mt., 05.04.2016., leg. \& det. N. Milosavljević; Niš (Pantelej), 04.2016., M.S.N. 157, leg. \& det. M. Kuštera.

60. Morchella semilibera DC

Beograd (Zvezdara), Ibrahim Hadžić, 18.04.1992., leg. \& det. I. Hadžić; Obedska bara (Debela gora), sub Mitrophora semilibera, Beronja 1995: 3; Beograd (Bežanija), 15.04.1995., leg. \& det. I. Hadžić; Fruška Gora Mt. (Stražilovo), sub Mitrophora semilibera, Karaman et al. 2012: 42; Kragujevac (Šumarice), 03.04.2009., leg. \& det. N. Milosavljević; Rudnik Mt. (Vraćevšnica monastery), 22.04.2012., leg. V. Milosavljević, det. N. Milosavljević; Knjaževac (Orešac vill.), 06.05.2013., leg. \& det. S. Stanojević; Suva planina Mt. (Ploče), 04.2015., leg. \& det. S. Lazarević; Goč Mt. (Lugareva kuća), 09.04.2015., leg. \& det. N. Milosavljević; Niš (Brzi brod), 30.03.2017., M.S.N. 172, leg. S. Lazarević, det. M. Kuštera.

\section{Morchella steppicola Zerova}

Novi Beograd, 27.03.1995., leg. \& det. I. Hadžić; Fruška Gora Mt. (Bukovac vill.), 17.04.2006., leg. \& det. D. Savić; Fruška Gora Mt. (Beočin), Perić \& Radić, 2013: 8; Niš (Cer hill), 03.2016., M.S.N. 124, leg. \& det. N. Minčić; Fruška Gora Mt. (Beočin), 12.03.2013. and 29.03.2018., F.G. 881, leg. \& det. D. Savić.

62.* Morchella tridentina Bres.

Avala Mt. (Šuplja stena), 10.05.2004., leg. \& det. I. Hadžić.

63. Verpa bohemica (Krombh.) J.Schrot.

Subotičko-horgoška peščara (Bukvać), Gajić 1986: 29; Beograd (Ada ciganlija), 23.03.1993., leg. \& det. I. Hadžić; Kragujevac (Šumarice), 16.03.2008., leg. \& det. N. Milosavljević; Fruška Gora Mt. (Popovica), sub Ptychoverpa bohemica, Karaman et al. 2012: 42; Fruška Gora Mt. (Popovica), 12.04.2013., leg. D.Grujić, det. D. Savić; Deliblatska peščara (Crni vrh), 10.04.2013., leg. \& det. D. Savić; Goč Mt. (Partizanske kolibe), 18.03.2015., leg. \& 
det. N. Milosavljević; Suva planina (Ploče), 04.2015., leg. \& det. I. Cvijetan; Niš (Popova glava), 03.2016., M.S.N. 219, leg. \& det. S. Lazarević; Šabac (Mali Zabran), 20.03.2016., leg. \& det. S. Stanojević.

\section{Verpa conica (Mull) Swartz}

Beograd (forest towards the aeroport Surčin), 04.1994., leg. \& det. I. Hadžić; Kragujevac (Metino brdo), 23.04.2009., leg. \& det. N. Milosavljević; Petrovaradinski rit, Karaman et al. 2012: 42; Rudnik Mt. (Dobrača vill.), 17.04.2013., leg. \& det. N. Milosavljević; Niš (Brzi Brod), 05.04.2018., M.S.N. 220, leg. \& det. S. Lazarević; Fruška Gora Mt. (Beočin), 23.04.2018., F.G. 899, leg. D. Grujić, det. D. Savić.

Fam.: Pezizaceae Dumort. 1829

65.*Adelphella babingtonii (Sacc.) Pfister., Matočec \& I.Kušan

Fruška Gora Mt. (Šakotinac), 09.09.2013., deciduous tree, rotten wood, leg. \& det. D. Savić; Goč Mt. (Cvetne livade), 15.07.2014., leg. \& det. N. Milosavljević; Fruška Gora Mt. (Rakovac vill.), 27.04.2015., deciduous tree, F.G. 376, leg. \& det. D. Savić; Kopaonik Mt. (Gobelja), 11.09.2015., Picea abies, cone, leg. \& det. I. Kajevska; Kopaonik Mt. (Gobelja), 05.09.2017., Picea abies, cone, F.G.706., leg. E. Bošković, det. D. Savić; Kukavica Mt. (Slatina vill.), 04.06.2017., on rotten wood, leg. \& det. S. Jovanović; Goč Mt. (Gajovača), 08.09.2017., leg. \& det. N. Milosavljević.

66.*Iodophanus carneus (Pers.) Korf.

Tomaševac vill., 07.03.2013., on sheep dung., leg. \& det. D. Savić; Fruška Gora Mt. (Grgurevci vill.), 07.03.2013., on sheep dung, leg. \& det. D. Savić; Fruška Gora Mt. (Brankovac), 01.06.2017., on horse dung, F.G. 626, leg. \& det. D. Savić; Fruška Gora Mt. (Glavica), 22.03.2018., on sheep dung, leg. \& det. D. Savić; Kopaonik Mt. (Jankove bare), 31.08.2018., on dung, F.G. 852 , leg. \& det. D. Savić.

67.*Iodophanus testaceus (Moug.) Korf.

Fruška Gora Mt. (Bukovac vill.), 12.06.2017., on soil, F.G. 646, leg. \& det. D. Savić.

68. Mattirolomyces terfezioides (Mattir.) E. Fisch.

Deliblatska peščara (UTM squares EQ16 and DQ98), sub Terfezia terfezioides, Ławrynowicz et al. 1997: 236; Deliblatska peščara 
(Devojački bunar /V. Lindtner, BEO/, Dragićev hat, Trujino naselje) and Subotička peščara (Ivančević 2016: 92).

69. Pachyphloeus citrinus Berk. \& Broome

Tara Mt. (Lokvica and Tisovo brdo-Lokvica) and Užice (Duboko vill.), Ivančević 2016: 96.

70.*Peziza ammophila Durieu \& Lév.

Deliblatska peščara, 25. 04.2008., on sandy soil, leg. \& det. B. Uzelac.

71.*Peziza ampliata Pers.

Kopaonik Mt. (Gobelja), 31.08.2018., on soil, F.G. 834, leg. \& det. D. Savić.

72. Peziza apiculata Cooke

Srbija, Karadžić 2003: 62, on Fagus sylvatica; Srbija, Karadžić \& Milijašević 2004: 28, on Fagus sylvatica.

73.*Peziza arvernensis Roze \& Boud.

Niš (Kamenički vis), 14.05.2016., on soil, leg. N. Minčić, det. I. Kajevska; Kukavica Mt. (Slatina vill.), 30.05.2017., on soil, F.G. 783, leg. S. Jovanović, det. D. Savić; Fruška Gora Mt. (Lipovača), 23.04.2018., F.G. 822, leg. S. Baluban, det. D. Savić.

74. Peziza badia Pers.

Fruška Gora Mt. (Glavica and Iriški venac), Karaman et al. 2012:

42; Fruška Gora Mt. (Brankovac), 08.07.2013., leg. \& det. D. Savić; Kukavica Mt. (Slatina vill.), 25.04.2017., on soil, F.G. 784, leg. S. Jovanović, det. D. Savić.

75. ${ }^{*}$ Peziza badiofusca (Boud.) Dennis

Niš (Knez vill.), 17.05.2017., on soil, M.S.N. 185, leg. M. Kuštera, det. I. Kajevska; Niš (Gabrovac vill.), 04.06.2017., on soil, M.S.N. 197, leg. \& det. I. Kajevska; Niš (Prosek vill.), on soil, 15.07.2018., M.S.N. 231, leg. \& det. I. Kajevska; Suva planina Mt. (Bojanine Vode), on soil, 03.08.2018., M.S.N. 252, leg. \& det. I. Kajevska; Kukavica Mt. (Slatina vill.), 30.05.2017., on sandy soil, F.G. 782, leg. S. Jovanović, det. D. Savić.

76. Peziza cerea Sow

Beograd (Ada ciganlija), 15.11.1992., leg. \& det. I. Hadžić; Fruška Gora Mt. (Popovica), Karaman et al. 2012: 42; Fruška Gora Mt. (Papratski do), 05.06.2012., leg. \& det. D. Savić; Fruška Gora Mt. (Stražilovo), 14.11.2012., on Acer campestre, rotten wood, leg. \& det. D. Savić; Suva planina Mt. (Ploče), on soil, 
10.07.2018., M.S.N. 238, leg. I. Cvijetan, det. I. Kajevska; Kopaonik Mt. (Jankove bare), 31.08.2018., leg. \& det. D. Savić.

77. ${ }^{*}$ Peziza depressa Pers.

Fruška Gora Mt. (Ravne), 07.06.2018., on soil, F.G. 825, leg. \& det. D. Savić; Fruška Gora Mt. (Paragovo), 08.06.2016., on soil, leg. \& det. D. Savić; Suva planina Mt. (Ploče), on soil, 09.07.2018., M.S.N. 237, leg. I. Cvijetan, det. I. Kajevska; Niš (Malča vill.), allong Nišava r., on soil, 27.07.2018., M.S.N. 244, leg. \& det. I. Kajevska; Suva planina Mt. (Gornja Studena vill.), on soil, 03.08.2018., M.S.N. 246, leg. \& det. I. Kajevska; Fruška Gora Mt. (Beočin monastery), 13.06.2018., on soil, F.G. 887, leg. \& det. D. Savić; Fruška Gora Mt. (Ledinci vill.), 20.07.2018., on soil, leg. \& det. D. Savić.

78. Peziza domiciliana Cooke

Beograd (Makiš), 23.04.1998., on Populus sp., leg. \& det. I. Hadžić; Kragujevac (Žeželj), 22.05.2014., leg. \& det. N. Milosavljević; Šabac (Gornja Vranjska), 24.10.2014. leg. \& det. S. Stanojević; Babušnica, 09.2015., lumber, leg. \& det. D. Stojanović; Niš (Pantelej), 18.09.2016., on crack from bacement, leg. \& det. I. Kajevska; Beograd (Botanical Garden), Vukojević et al. 2016: 253; Fruška Gora Mt. (Beočin), 12.04.2017., in basement, F.G. 543, leg. D. Grujić, det. D. Savić.

79. Peziza echinospora P.Karst.

Fruška Gora Mt. (Iriški venac), Karaman et al. 2012: 42.

80. Peziza fimeti (Fuckel) E.C.Hansen

Fruška Gora Mt. (Ravne), 14.05.2013., Cervus elaphus, dung, leg. $\&$ det. D. Savić.

\section{Peziza granularis Donadini}

Fruška Gora Mt. (Andrevlje), 30.10.2012., on soil, leg. \& det. D. Savić; Fruška Gora Mt. (Erdelj), 09.11.2015., on soil, F.G. 329, leg. \& det. D. Savić; Niš (Brzi brod), 03.11.2015., on sandy soil, M.S.N. 128, leg. \& det. I. Kajevska; Fruška Gora Mt. (Brankovac), 16.05.2017., on soil, F.G. 647, leg. \& det. D. Savić.

\section{2.*Peziza micropus Pers.}

Fruška Gora Mt. (Iriški venac), 12.07.2016., on rotten wood, leg. $\&$ det. D. Savić. 
83.*Peziza michelii (Boud.) Dennis

Obrenovac (Duboka), 22. VI 2004., leg. \& det. B. Uzelac; Fruška Gora Mt. (Paragovo), 03.07.2018., on soil, F.G. 884, leg. \& det. D. Savić.

84.*Peziza petersii Berk.

Avala Mt., 04.07.2005., on a burnt wood, leg. \& det. B. Uzelac.

85. Peziza phyllogena Cooke

Beograd (Lipovička šuma), 04.05.1997., leg. \& det. I. Hadžić; Kragujevac (Žeželj), 09.05.2014., leg. \& det. N. Milosavljević; Niš (Popova glava), on soil, M.S.N. 116, Kajevska et al. 2018: 82.

86. Peziza repanda Wahlenb

Beograd (Košutnjak), sub Plicaria repanda, Ranojević 1910: 352; Deliblatska peščara, 27.04.1998., leg. \& det. I. Hadžić; Fruška Gora Mt. (Bukovac vill.), Karaman et al. 2012: 42; Goč Mt. (Bela reka), 22.05.2014., leg. \& det. N. Milosavljević; Suva planina (Miljkovac vill.), 23.10.2016., on rotten log, M.S.N. 116, leg. \& det. I. Kajevska; Suva planina Mt. (Ćelije vill.), 01.05.2017., on soil and rotten wood, M.S.N. 174, leg. \& det. I. Kajevska; Bukulja Mt., 20.04.2018., on sawdust, leg. \& det. S. Stanojević.

87.*Peziza saniosa Schrad.

Babušnica (Vava vill.), 07.2011., on soil, leg. \& det. D. Stojanović; Goč Mt. (Brezjak), 08.06.2014., leg. \& det. N. Milosavljević; Brus (Tršanovci vill.), 19.06.2016., on soil, leg. \& det. I. Kajevska; Niš (Knez vill.), 17.05.2017., on soil, M.S.N. 184, leg. $\&$ det. I. Kajevska; Kukavica Mt. (Slatina vill.), 11.05.2017., on soil, F.G. 786, leg. S. Jovanović, det. D. Savić; Fruška Gora Mt. (Papratski do), 02.06.2017., on soil, F.G. 652, leg. \& det. D. Savić; Niš (Prosek vill.), on soil, 15.07.2018., M.S.N. 230, leg. \& det. I. Kajevska; Suva planina Mt. (Bojanine vode), on soil, 03.08.2018., M.S.N. 251, leg. \& det. I. Kajevska; Fruška Gora Mt. (Paragovo), 03.07.2018., on soil, F.G. 895, leg. \& det. D. Savić.

88. Peziza subisabellina Le Gal ex M.M. Moser

Fruška Gora Mt. (Ravne), 05.06.2018., F.G. 872, leg. \& det. D. Savić.

89. Peziza succosa Berk.

Beograd (Zvezdara), 23.05.1989., leg. \& det. I. Hadžić; Kragujevac (Žeželj), 18.05.2014., leg. \& det. N. Milosavljević; Niš (Cerje vill.), 17.11.2015., on soil, leg. \& det. I. Kajevska; Fruška Gora Mt. (Iriški venac), 09.07.2018., on soil, F.G. 868, leg. \& det. 
D. Savić; Fruška Gora Mt. (Paragovo), 05.07.2018., on soil, leg. \& det. D. Savić; Fruška Gora Mt. (Ledinci), 20.07.2018., on soil, F.G. 898, leg. \& det. D. Savić; Suva planina Mt. (Bojanine Vode), on soil, 03.08.2018., M.S.N. 250, leg. \& det. I. Kajevska.

90.*Peziza succosella (Le Gal \& Romagn.) M.M. Moser ex Aviz.Hersh. \& Nemlich

Fruška Gora Mt. (Grgeteg), 21.08.2018., on soil, F.G. 805, leg. \& det. D. Savić.

\section{Peziza tenacella W.Phillips}

Tara Mt. (Crveni potok), sub Plicaria violacea, Čolić 1967: 411; Beograd (Makiš), 22.05.2011., leg. \& det. I. Hadžić; Goč Mt. (Golići), 11.06.2013., leg. \& det. N. Milosavljević; Goč Mt. (Ovčarnik), 13.10.2014., N.M. 141013-1, leg. \& det. N. Milosavljević.

92.*Peziza varia (Hedw.) Alb. \& Schwein.

Fruška Gora Mt. (Paragovo), 04.11.2013., leg. \& det. D. Savić; Niš (Gabrovac vill.), 30.06.2016., on soil with woody debris, leg. \& det. I. Kajevska; Goč Mt. (Brezna vill.), 09.10.2016., leg. \& det. N. Milosavljević; Kukavica Mt. (Slatina vill.), 01.05.2017., on soil with woody debris, leg. \& det. S. Jovanović.

93. Peziza vesiculosa Bull

Beograd (Topčider), sub Pustularia vesiculosa, Ranojević 1910: 352 and Ranojević 1937: 5; Fruška Gora Mt. (Brankovac), 13.11.2013., on horse dung, leg. \& det. D. Savić; Fruška Gora Mt. (Iriški venac and Sremska Kamenica), Karaman et al. 2012: 42; Kragujevac (Žeželj), 09.05.2014., leg. \& det. N. Milosavljević; Kukavica Mt. (Slatina vill.), 25.04.2017., on the straw, F.G. 785, leg. S. Jovanović, det. D. Savić.

94. Sarcosphaera coronaria (Jacq.) J. Schröt.

Suva planina Mt. (Ploča), 04.2014., half-buried in soil, leg. \& det. M. Kuštera; Stara planina Mt., Sadiković \& Kuštera 2013: 38; Niš (Knez vill.), 04.2014., half-buried in soil, leg. \& det. M. Kuštera; Babušnica (Vava vill.), 04.2014., half-buried in soil, leg. \& det. D. Stojanović; Dimitrovgrad (Slivnica vill.), 13.04.2018, F.G. 791, leg. M. Petrov, det. D. Savić.

95. Terfezia arenaria (Moris) Trappe

Deliblatska peščara, 25.07.1999., leg. \& det. I. Hadžić; Svrljig, Ivančević 2016: 105. 
Fam.: Pyronemataceae Corda 1842

96. Aleuria aurantia (Pers.) Fuckel

Rakovica, Ranojević 1910: 352; Fruška Gora Mt., sub Peziza aurantia, Ranković 1955: 4; Tara Mt. (Crveni potok), sub Peziza aurantia, Čolić 1967: 411; Zasavica (Vrbovac, Preseka, Trebljevine, Batar, Prekojovača and Šumareva ćuprija), Cviyanovicy et al. 2009: 237; Babušnica (Vava vill.), 10.2009., on soil, leg. \& det. D. Stojanović; Fruška Gora Mt. (Iriški venac), Karaman et al. 2012: 42; Fruška Gora Mt. (Papratski do), 21.11.2012., on soil, leg. \& det. D. Savić; Goč Mt. (Brezna vill.), 25.09.2013., leg. \& det. N. Milosavljević; Palićko jezero, 30.01.2015., F.G.72, leg. J. Došai, det. D. Savić; Kopaonik Mt. (Markov kamen), 01.09.2015., on soil, leg. M. Kuštera, det. I. Kajevska; Cer Mt. (Radovašnica), 18.10.2015., on soil, leg. \& det. S. Stanojević; Niš (Knez vill.), 25.10.2015., on soil, leg. \& det. I. Kajevska; Kukavica Mt. (Slatina vill.), 28.09.2016., on soil, leg. \& det. S. Jovanović; Niš (Gradac), 18.10.2016., on soil, M.S.N. 120, leg. S. Lazarević, det. I. Kajevska; Vlasotince (Brezovica vill.), 29.10.2016., on soil, leg. S. Lazarević, det. I. Kajevska; Kopaonik Mt. (Sunčani vrhovi), 01.09.2018., on soil, leg. \& det. D. Savić.

97.*Aleuria boudieri (Höhn.) J. Moravec

Fruška Gora Mt. (Paragovo), 07.07.2016., on soil, F.G. 477, leg. $\&$ det. D. Savić.

98. Aleuria marchica (Rehm) Boud.

Avala Mt., sub Aleurina marchica, on Fagus sylvatica, Ranojević 1910: 352; Krepoljin (Komnenski potok), sub Aleurina marchica, on Fagus sylvatica, Ranojević 1910: 352.

99.*Aleurina tenuiverrucosa Dougoud \& Roffler

Despotovac (Sladaja vill.), 24.07.2013. leg. \& det. Ž. Jorgovanović; Fruška Gora Mt. (Stražilovo), 24.08.2016., on soil, F.G.491, leg. \& det. D. Savić; Krupanj, 25.12.2017., F.G.726, leg. M. Marković, det. D. Savić.

100.*Anthracobia melaloma (Alb. \& Schwein.) Boud.

Beograd (Jabučki rit), 18. 11. 2000., leg. \& det. I. Hadžić; Fruška Gora Mt. (Susek vill.), 30.11.2012. leg. \& det. D. Savić; Fruška Gora Mt. (Stražilovo), 10.06.2013., on burned ground, F.G. 235, leg. \& det. D. Savić; Fruška Gora Mt. (Iriški venac), 12.06.2013., on burned ground, leg. \& det. D. Savić; Goč Mt. (Ovčarnik), 13.10.2014., leg. \& det. N. Milosavljević. 
101.*Byssonectria deformis (P. Karst.) U. Lindem. \& M. Vega Fruška Gora Mt. (Stražilovo), 21.07.2016., deciduous tree, rotten wood, F.G. 493, leg. \& det. D. Savić; Fruška Gora Mt. (Paragovo), 09.05.2018., deciduous tree, F.G. 848, leg. \& det. D. Savić.

102. Cheilymenia granulata (Bull.) J. Moravec

Goč Mt. (Cvetne livade), 10.06.2014., leg. \& det. N. Milosavljević; Kopaonik Mt. (Gobelja), 11.09.2015., on cow dung, M.S.N. 218, leg. \& det. I. Kajevska; Beograd (Botanical Garden), Vukojević et al. 2016: 251; Kopaonik Mt. (Gobelja), 01.09.2018., on cow dung, F.G. 826, leg. \& det. D. Savić.

103.* Cheilymenia rubra (Roum.) Boud.

Fruška Gora Mt. (Stražilovo), 06.06.2014., on dung, leg. \& det. D. Savić; Jagodina, 17.04.2018., on chicken dung, F.G. 880, leg. J. Biserčić, det. D. Savić.

104.*Cheilymenia stercorea (Pers.) Boud.

Fruška Gora Mt. (Brankovac), 20.10.2015., on horse dung, F.G. 299, leg. \& det. D. Savić; Suva Planina Mt. (road from Bojanine Vode to mountain hut), 22.08.2017., on cow dung, M.S.N. 203, leg. \& det. I. Kajevska.

105.* Cheilymenia theleboloides (Alb. \& Schwein.) Boud.

Goč Mt. (Brezjak), 02.11.2014., leg. \& det. N. Milosavljević.

106. ${ }^{*}$ Cheilymenia vitellina (Pers.) Dennis

Goč Mt. (Cvetne livade), 01.07.2014., leg. \& det. N. Milosavljević; Fruška Gora Mt. (Stražilovo), 10.09.2014., on soil, F.G. 197 and 16.09.2016., on soil, F.G. 564, leg. \& det. D. Savić; Fruška Gora Mt. (Grgurevci vill.), 18.10.2017., on soil, F.G. 723, leg. \& det. D. Savić.

107. Genea fragrans (Wallr.) Sacc.

Užice (Duboko vill.) and Obrenovac (Urovci vill.), Ivančević 2016: 79 .

108. Genea klotzschii Berk. \& Broome

Beograd (Makiš), 28.09.1997., leg. \& det. I. Hadžić; Beograd (Bojčinska šuma), 28.09.1997., leg. \& det. I. Hadžić; Beograd (Bojčinska šuma), Ivančević \& Davidović 2011: 24; Beograd (Makiš), Ivančević 2016: 80.

109. Genea sphaerica Tul. \& C. Tul.

Valjevo (Popučke), Ivančević 2016: 81. 
110. Genea vagans Mattir.

Koritnjak Mt. (Koritnik vill.) and Valjevo (Popare), Ivančević 2016: 81.

111. Genea verrucosa Vittad.

Koviljsko-Petrovaradinski rit, Katanić et. al. 2015: 331; Lajkovac (Bajevac vill. and Jabučje vill.), Ivančević 2016: 82.

112.*Geopora arenicola (Lév.) Kers

Fruška Gora Mt. (Stražilovo), 10.06.2013., on soil, F.G. 203, leg. $\&$ det. D. Savić.

113.*Geopora foliacea (Schaeff.) S. Ahmad

Fruška Gora Mt. (Beočin), 25.03.2016., on soil, F.G. 394, leg. \& det. D. Savić.

114.*Geopora sumneriana (Cooke) M. Torre

Niš (Gornja Toponica vill.), 03.2014., soil, semi buried, M.S.N. 149; leg. \& det. N. Minčić; Kragujevac (Bubanj), 29.03.2014., leg. \& det. N. Milosavljević; Fruška Gora Mt. (Sremska Kamenica), 29.03.2016., under Cedrus deodara, leg. \& det. D. Savić; Novi Sad, 29.03.2016., under Cedrus deodara, F.G.428; Niš (Pantelej), 08.05.2017., on soil, under planted Cedrus atlantica, M.S.N. 183; leg. \& det. M. Kuštera.

115.*Geopora tenuis (Fuckel) T.Schumach.

Fruška Gora Mt. (Petrovaradin), 13.09.2013., on soil, leg. \& det. D. Savić.

116.* Geopyxis carbonaria (Alb. \& Schwein.) Sacc.

Bajina Bašta (Dub vill.), 23. 04 2001., burned place in a pine forest, leg. \& det. B. Uzelac; Tara Mt. (Kriva jela), 17.06.2003., burned place in a spruce and fir forest, leg. \& det. B. Uzelac; Goč Mt. (Cvetne livade), 06.08.2014., N.M. 140608-1, leg. \& det. N. Milosavljević.

117. Humaria hemisphaerica (Wigg.) Fuckel

Avala Mt., sub Lachnea hemisphaerica, Ranojević 1910: 352; Stara planina Mt. (Dojčinovo vrelo-Jabučko ravnište and Babin zub-Topli do), Ivančević \& Beronja 2007: 16; Babušnica (Vava vill.), 07.2009., on soil, leg. \& det. D. Stojanović; Cer Mt. (Tekeriš), 09.10.2010., leg. \& det. I. Hadžić; Beograd (Bojčinska šuma), Ivančević \& Davidović 2011: 25; Fruška Gora Mt. (Paragovo), Karaman et al. 2012: 42; Fruška Gora Mt. (Stražilovo), 04.07.2013., on soil, leg. \& det. D. Savić; Goč Mt. (Cvetne 
livade), 14.07.2014., leg. \& det. N. Milosavljević; Brus (Tršanovci vill.), 19.06.2016., on soil, leg. \& det. I. Kajevska; Niš (Kamenički vis), 15.10.2016., on soil, leg. \& det. I. Kajevska; Kukavica Mt. (Slatina vill.), 29.05.2017., on soil, leg. \& det. S. Jovanović; Fruška Gora Mt. (Beočin monastery), 04.07.2018., on soil, leg. \& det. D. Savić; Fruška Gora Mt. (Iriški venac), 12.07.2018., on soil, F.G. 882, leg. \& det. D. Savić.

118. *Humaria solisequia (Quel.) Van Vooren \& Moyne

Fruška Gora Mt. (Iriški venac), 06.11.2008., on soil, leg. \& det. D. Savić.

119. Hydnocystis bombycina (Vittad.) Healy \& M.E. Sm.

Svrljig (Guševac vill.), Suva planina Mt. (Krastavče vill.), Koritnjak Mt. (Koritnik vill.), Tara Mt. (Mitrovac) and Žitorađa (Dubovo vill.), Ivančević 2016: 104.

Note: Finding and the material form Guševac (form1992) was discussed in Kumar et al. 2017: 13 but the locality was cited wrongly as Grisevac.

120.*Lamprospora carbonicola Boud.

Fruška Gora Mt. (Erdelj), 20.10.2015., on Funaria hygrometrica, F.G. 335, leg. \& det. D. Savić.

\section{1.*Lamprospora miniata De Not.}

Fruška Gora Mt. (Čerević vill.), 20.10.2016., on Pottia lanceolata, F.G. 530, leg. \& det. D. Savić; Fruška Gora Mt. (Bukovac vill.), 19.01.2018., on Pottia lanceolata, F.G. 607, leg. \& det. D. Savić.

122.*Leucoscypha leucotricha (Alb. \& Schwein.) Boud.

Fruška Gora Mt. (Grgeteg vill.), 08.06.2012., on soil, leg. \& det. D. Savić; Fruška Gora Mt. (Stražilovo), 02.07.2015., on soil, F.G. 283, leg. \& det. D. Savić.

123.*Melastiza contorta (Massee \& Crossl.) Spooner \& Y.J. Yao Goč Mt. (Klupica), 17.10.2014., leg. \& det. N. Milosavljević.

124. Melastiza cornubiensis (Berk. \& Broome) J. Moravec Stara planina Mt. (Babin zub-Dojčinovo vrelo), sub Melastiza chateri, Ivančević \& Beronja 2007: 16; Fruška Gora Mt. (Erdelj), 18.11.2015., on soil, F.G. 397, leg. \& det. D. Savić.

125.*Melastiza flavorubens (Rehm) Pfister \& Korf

Fruška Gora Mt. (Popovica), 05.10.2015., on soil, F.G. 276, leg. \& det. D. Savić; Brus, 03.09.2016, leg. \& det. M. Slavova. 
126.*Miladina lecithina (Cooke) Svrček

Fruška Gora Mt. (Vrdnik vill.), 07.08.2013., deciduous tree, rotten wood, leg. \& det. D. Savić; Fruška Gora Mt. (Paragovo), 01.09.2013. leg. \& det. D. Savić; Fruška Gora Mt. (Paragovo), 11.07.2016., F.G. 455, leg. \& det. D. Savić.

127.*Neottiella albocincta (Berk. \& M.A. Curtis) Sacc.

Fruška Gora Mt. (Stražilovo), 19.08.2014., on Atrichum undulatum, F.G. 57, leg. \& det. D. Savić; Niš (Gabrovac vill.), 26.05.2016., on A. undulatum, M.S.N. 123, leg. V. Lilić, det. I. Kajevska; Brus (Tršanovci vill.), 19.06.2016., on A. undulatum, M.S.N. 165, leg. \& det. I. Kajevska.

128. Neottiella vivida (Nyl.) Dennis

Vlasotince (Brezovica vill.), 29.10.2016., on moss, M.S.N. 103, leg. S. Lazarević, det. M. Kuštera; Svrljig (Grbavče vill.), on Polytrichum sp., M.S.N. 101, Kajevska et al. 2018: 82; Niš (Gradac), on moss, M.S.N. 102, Kajevska et al. 2018: 82; Vlasina (UTM squares FN02), 30.07.2018., on Polytrichum piliferum, leg. N. Marić, det. D. Savić.

129.*Octospora axillaris (Nees) M.M. Moser

Fruška Gora Mt. (Sremska Kamenica), 08.01.2018., on Phascum cuspidatum, F.G. 604, leg. \& det. D. Savić.

130. Octospora erzbergeri Benkert

Đerdap National Park (Ploče), on Pseudoleskeella nervosa, Benkert 2006: 2.

131.*Octospora gemmicola Benkert

Fruška Gora Mt. (Erdelj), 20.10.2015., on Bryum sp., F.G. 352, leg. \& det. D. Savić; Fruška Gora Mt. (Erdelj), 18.01.2018., on Bryum capillare, F.G. 605, leg. \& det. D. Savić; Fruška Gora Mt. (Bukovac vill.), 19.01.2018., on Bryum caespiticium, F.G. 606, leg. \& det. D. Savić.

132.* Octospora gyalectoides Svrček \& Kubička

Fruška Gora Mt. (Brankovac), 26.12.2013., on Barbula unguiculata, leg. \& det. D. Savić; Fruška Gora Mt. (Rakovac vill.), 20.02.2014., on Pottia sp., leg. \& det. D. Savić; Fruška Gora Mt. (Sremska Kamenica), 08.01.2018., on Pottia sp., F.G. 603, leg. \& det. D. Savić. 
133.* Octospora leucoloma Hedw.

Fruška Gora Mt. (Brankovac), 26.12.2013., on Bryum argenteum, leg. \& det. D. Savić; Fruška Gora Mt. (Jabuka), 11.10.2013., on Bryum argenteum, leg. \& det. D. Savić.

\section{4.*Octospora musci-muralis Graddon}

Fruška Gora Mt. (Sremska Kamenica), 03.03.2014., on Grimmia pulvinata, leg. \& det. D. Savić; Niš (Prosek vill.), 26.12.2017., on Grimmia pulvinata., M.S.N. 215, leg. \& det. I. Kajevska; Fruška Gora Mt. (Ravne), 24.01.2018., on Grimmia pulvinata, F.G. 602, leg. \& det. D. Savić.

135. Octospora pannosa T. Richter, M. Vega \& D. Savić

Fruška Gora Mt. (Paragovo), on Brachytheciastrum velutinum, Vega et al. 2018: 1004.

136. Octospora phagospora (Flageolet \& Lorton) Dennis \& Itzerott

Đerdap National Park (Šomrdski kamen), Benkert 2007: 17.

137.* Octospora wrightii (Berk. \& M.A. Curtis) J. Moravec

Fruška Gora Mt. (Paragovo), 12.01.2018., on Amblystegium serpens, F.G. 737, leg. \& det. D. Savić.

138. Otidea alutacea Pers.

Tara Mt. (Crveni potok), Čolić 1967: 411; Fruška Gora Mt. (Sremska Kamenica), sub Otidea cohleata $\beta$ alutacea, Schulzer et al. 1866: 62; Fruška Gora Mt. (Grgeteg vill.), Karaman et al. 2012: 42, on soil, leg. \& det. D. Savić; Fruška Gora Mt. (Vrdnik vill.), 31.10.2013., on soil, leg. \& det. D. Savić; Goč Mt. (Ovčarnik), 17.10.2014., leg. \& det. N. Milosavljević; Niš (above Gradac and Knez vill.), 06.10.2015., M.S.N. 129, leg. \& det. I. Kajevska; Niš (Kamenički vis), 15.10.2016., leg. \& det. I. Kajevska; Fruška Gora Mt. (Crveni čot), 19.07.2018., on soil, F.G. 833, leg. \& det. D. Savić; Kopaonik Mt. (Jankove bare), 31.08.2018., on soil, F.G. 827, leg. \& det. D. Savić.

139.*Otidea brunneoparva Harmaja ex K. Hansen, M. Carbone, Olariaga \& Van Vooren

Goč Mt. (Prerovo), 12.08.2018., leg. \& det. N. Milosavljević.

140.* Otidea bufonia (Pers.) Boud.

Beograd (Bojčinska šuma), 03.10.2010., leg. \& det. I. Hadžić; Niš (Gradac), 2013., leg. \& det. M. Kuštera; Goč Mt. (Bela reka), 24.10.2014., leg. \& det. N. Milosavljević. 
141. Otidea cochleata (L.) Fuckel

Obedska bara, 21.10.1998., leg. \& det. I. Hadžić; Beograd (Bojčinska šuma), Ivančević \& Davidović 2011: 25.

142.* Otidea concinna (Pers.) Sacc.

Cer Mt. (Tekeriš), 09.10.2010., leg. \& det. I. Hadžić.

143. Otidea leporina (Batsch) Fuckel

Tara Mt. (Crveni potok), Čolić 1967: 411; Goč Mt. (Brezjak), 04.10.2014., leg. \& det. N. Milosavljević.

144. *Otidea mirabilis Bolognini \& Jamoni

Goč Mt. (Brezna), 29.10.2015., N.M. 151029-1, leg. \& det. N. Milosavljević; Goč Mt. (Cvetne livade), 26.07.2018., leg. \& det. N. Milosavljević.

145. Otidea onotica (Pers.) Fuckel

Tara Mt. (Crveni potok), Čolić 1967: 411; Fruška Gora Mt. (Paragovo), Karaman et al. 2012: 42, leg. \& det. D. Savić; Fruška Gora Mt. (Vrdnik vill.), 01.10.2014., on soil, F.G. 71, leg. \& det. D. Savić; Goč Mt. (Prerovo), 09.10.2014., leg. \& det. N. Milosavljević; Niš (Gradac), on soil, 06.10.2015., M.S.N. 129, leg. \& det. I. Kajevska; Kukavica Mt. (Slatina vill.), 29.09.2016., on soil, leg. \& det. S. Jovanović; Goč Mt. (Cvetne livade), 09.10.2017., N.M. 171009-2, leg. \& det. N. Milosavljević; Petrovac na Mlavi (Melnica vill.), on soil, 12.07.2018., leg. D. Lazić, det. D. Savić; Kopaonik Mt. (Jankove bare), 31.08.2018., on soil, F.G. 866, leg. \& det. D. Savić; Jelova Gora Mt. (Gostinica vill.), 03.08.2018., F.G. 812, leg. M. Adžić, det. D. Savić.

146.*Otidea subformicarum Olariaga, Van Vooren, Carbone \& K. Hansen

Goč Mt. (Brezjak), 05.08.2014., N.M. 140805-1, leg. \& det. N. Milosavljević.

147.*Parascutellinia carneosanguinea (Fuckel) T.Schumach.

Despotovac (Vodna vill.,), 29.07.2013., along r.Resava, leg. \& det. Ž. Jorgovanović; Sićevačka klisura, on soil, aluvial place, among algae, moss and rootlets, 30.10.2018., leg. \& det. I. Kajevska.

148.*Pseudombrophila cervaria (W.Phillips) Brumm.

Fruška Gora Mt. (Petrovaradin), 13.09.2013., Capreolus capreolus, dung, leg. \& det. D. Savić; Fruška Gora Mt. (Brankovac), 16.05.2017., dung, leg. \& det. D. Savić. 
149.*Pseudombrophila theioleuca Rolland

Fruška Gora Mt. (Iriški venac), 08.06.2018., on manured soil, leg. $\&$ det. D. Savić.

150.*Pulvinula convexella (P.Karst.) Pfister

Fruška Gora Mt. (Astal), 12.09.2014., on burned ground, F.G. 76, leg. \& det. D. Savić; Fruška Gora Mt. (Rakovac vill.), 07.07. 2016., on soil, F.G. 478, leg. \& det. D. Savić.

151.*Pyronema domesticum (Sowerby) Sacc.

Fruška Gora Mt. (Iriški venac), 12.06.2013., on burned wood, F.G. 239, leg. \& det. D. Savić; Fruška Gora Mt. (Zmajevac), 28.08.2013., on burned wood, leg. \& det. D. Savić; Suva planina Mt. (Bojanine vode), 22.08.2017., on burned wood, M.S.N. 201, leg. \& det. I. Kajevska.

152. Pyronema omphalodes (Bull.) Fuckel

Fruška Gora Mt. (Sremska Kamenica), sub Pyronema marianum, Schulzer et al 1866: 61; Fruška Gora Mt. (Iriški venac), 14.06. 2013., on burned wood, leg. \& det. D. Savić.

153. Scutellinia crinita (Bull.) Lambotte

Goč Mt. (Dobre vode), trunk, Matočec et al. 1995: 14; Fruška Gora Mt. (Paragovo), 30.01.2014., Acer campestre, bark, leg. \& det. D. Savić; Fruška Gora Mt. (Stražilovo), 23.05.2014., dung, F.G. 103, leg. \& det. D. Savić; Fruška Gora Mt. (Ravne), 02.05.2014., F.G. 259, leg. \& det. D. Savić; Fruška Gora Mt. (Zmajevac), 05.06.2017., F.G. 687, leg. \& det. D. Savić; Suva planina Mt. (Gadžin han), 01.06.2017., on deciduous tree, branch, M.S.N. 193, leg. \& det. I. Kajevska.

154. Scutellinia legaliae Lohmeyer \& Häffner

Niš (Gabrovac vill.), on soil, M.S.N. 199, Kajevska et al. 2018: 83

155.*Scutellinia minor (Velen.) Svrček

Fruška Gora Mt. (Erdelj), 27.06.2013., on soil, leg. \& det. D. Savić; Fruška Gora Mt. (Paragovo), 05.10.2016., on soil, F.G.466, leg. \& det. D. Savić.

\section{6. *Scutellinia nigrohirtula (Svrček) Le Gal}

Fruška Gora Mt. (Stražilovo), 25.08.2016., rotten wood, F.G. 467, leg. \& det. D. Savić; Fruška Gora Mt. (Papratski do), 30.08.2016., rotten wood, F.G. 471, leg. \& det. D. Savić; Kopaonik Mt. (Gvozdac), 05.09.2017., on Picea abies, cone, F.G.707, leg. E. 
Bošković, det. D. Savić; Niš (Malča vill.), on soil, wet place, 22.10.2018., leg. \& det. I. Kajevska.

157. ${ }^{*}$ Scutellinia olivascens (Cooke) Kuntze

Kopaonik Mt. (road to Raška, under Hotel Putnik), 28.07.2017., on wet soil, leg. S. Vlahović-Nikolić, det. I. Kajevska.

158. Scutellinia scutellata (L.) Lambotte

Rakovica, sub Lachnea scutellata, Ranojević 1910: 352; Obedska bara (Matijevica-Kadionica), Beronja 1995: 3; Stara planina Mt. (Babin zub-Dojčinovo vrelo, Dojčinovo vrelo-Jabučko ravnište and Jabučko ravnište), Ivančević \& Beronja 2007: 16; Beograd (Bojčinska šuma), Ivančević \& Davidović 2011: 26; Fruška Gora Mt. (Dumbovo, Glavica, Iriški venac, Letenka, Paragovo and Vrdnik vill.), Karaman et al. 2012: 42; Fruška Gora Mt. (Testera), 30.05.2013., F.G. 190, leg. \& det. D. Savić; Fruška Gora Mt. (Stražilovo), 10.06.2013., F.G. 240, leg. \& det. D. Savić; Goč Mt. (Cvetne livade), 17.07.2014., leg. \& det. N. Milosavljević; Kukavica Mt. (Slatina vill.), 19.05.2017., on wood, leg. \& det. S. Jovanović; Kopaonik Mt. (road to Raška, under Hotel Putnik), 28.07.2017., on deciduous tree, branch, leg. \& det. I. Kajevska.

159. * Scutellinia setosa (Nees) Kuntze

Fruška Gora Mt. (Rakovac vill.), 16.07.2014., rotten wood, leg. \& det. D. Savić; Goč Mt. (Bela reka), 17.10.2014., leg. \& det. N. Milosavljević; Niš (Donja Vrežina vill.), 01.07.2015., on Salix sp., rotten wood, M.S.N. 175, leg. \& det. I. Kajevska; Fruška Gora Mt. (Stražilovo), 25.08.2016., rotten wood, F.G. 627, leg. \& det. D. Savić.

\section{0.*Scutellinia subhirtella Svrček}

Fruška Gora Mt. (Rakovac vill.), 06.07.2017., on soil, under Phragmites australis, F.G. 653, leg. \& det. D. Savić; Fruška Gora Mt. (Bešenovački Prnjavor vill.), 22.07.2017., on soil, F.G. 618, leg. \& det. D. Savić; Tomaševac, 20.08.2018., on soil, under Phragmites australis).

161. Scutellinia trechispora (Berk. \& Broome) Lambotte

Goč Mt. (Bela reka), 14.07.2014., leg. \& det. N. Milosavljević; Brus (Tršanovci vill.), 19.06.2016., on soil, M.S.N. 164, leg. I. Cvijetan, det. I. Kajevska; Suva planina Mt. (Gadžin han), on soil, M.S.N. 213, Kajevska et al. 2018: 83; Kukavica Mt. (Slatina vill.), 19.05.2017., F.G. 788, leg. S. Jovanović, det. D. Savić. 
162. Scutellinia umbrorum (Fr.) Lambotte

Beograd (Bojčinska šuma), 24.10.2010., leg. \& det. I. Hadžić; Fruška Gora Mt. (Stražilovo), Karaman et al. 2012: 42; Goč Mt. (Cvetne livade), 14.07.2014., N.M. 140714-4, leg. \& det. N. Milosavljević; Sićevačka klisura (Kunovica vill.), 02.07.2015., on soil, M.S.N. 176, leg. \& det. I. Kajevska; Fruška Gora Mt. (Papratski do), 16.06.2017., on soil, F.G. 643, leg. \& det. D. Savić.

163. * Sepultariella semi-immersa (P.Karst) Kutorga

Fruška Gora Mt. (Stražilovo), 02.11.2015., on soil, F.G. 328, leg. \& det. D. Savić; Fruška Gora Mt. (Erdelj), 09.11.2015., on soil, F.G. 345, leg. \& det. D. Savić; Niš (Malča vill., allong Nišava r.), on soil, 27.07.2018., M.S.N. 240, leg. \& det. I. Kajevska.

164. Sowerbyella fagicola J. Moravec

Suva planina Mt. (Gadžin Han), 29.05.2016/18., on deciduous tree, branch, M.S.N. 162, 239, Kajevska \& Savić (in press).

165. * Sowerbyella rhenana (Fuckel) J. Moravec

Suva planina (Jelovica vill.), 11.07.2018., mixed forest, on soil, leg. G. Taskov, det. D. Savić.

166. Tarzetta catinus (Holmsk.) Korf \& J.K.Rogers

Beograd (Ada ciganlija), 24.09.1996., leg. \& det. I. Hadžić; Goč Mt. (Cvetne livade), 12.06.2014., leg. \& det. N. Milosavljević; Fruška Gora Mt. (Stražilovo), Karaman et al. 2012: 42; Fruška Gora Mt. (Stražilovo), 16.10.2015., on soil, leg. \& det. D. Savić; Niš (Popova glava), 14.05.2016., on soil, M.S.N. 121, leg. \& det. I. Kajevska; Fruška Gora Mt. (Iriški venac), 24.05.2014., on soil, F.G. 243, leg. \& det. D. Savić; Kukavica Mt. (Slatina vill.), 26.04.2017., on soil, leg. \& det. S. Jovanović.

167. Tarzetta cupularis (L.) Svrček

Beograd (Bojčinska šuma), 13.10.1996., leg. \& det. I. Hadžić; Beograd (Bojčinska šuma), Ivančević \& Davidović 2011: 26; Fruška Gora Mt. (Andrevlje), 05.09.2013., on soil, leg. \& det. D. Savić; Fruška Gora Mt. (Paragovo), 25.09.2013., on soil, leg. \& det. D. Savić; Fruška Gora Mt. (Ravne), 07.04.2014., on soil, F.G. 157, leg. \& det. D. Savić; Šabac (Mali Zabran), 28.04.2016., on soil, leg. \& det. S. Stanojević; Goč Mt. (Cvetne livade), 28.08. 2014., leg. \& det. N. Milosavljević; Suva planina Mt. (Gadžin han), 29.05.2016., on soil, M.S.N. 144, leg. \& det. I. Kajevska; Niš (Gabrovac vill.), 04.06.2017., on soil, M.S.N. 196, leg. \& det. I. Kajevska; Bukulja Mt., 20.04.2018., on soil, leg. \& det. S. 
Stanojević; Fruška Gora Mt. (Stražilovo), 15.05.2018., on soil, F.G. 870, leg. \& det. D. Savić; Fruška Gora Mt. (Crveni čot), 18.07.2018., on soil, leg. \& det. D. Savić.

168.*Tricharina praecox (P.Karst.) Dennis

Fruška Gora Mt. (Stražilovo), 10.06.2013., on soil, F.G. 183, leg. $\&$ det. D. Savić.

169.*Trichophaea gregaria (Rehm) Boud.

Kopaonik Mt. (Jankove bare), 31.08.2018., on soil, F.G. 839, leg. $\&$ det. D. Savić.

170.* Trichophaea hemisphaerioides (Mouton) Graddon

Goč Mt. (Arboretum), 19.09.2014., N.M. 140714-3, leg. \& det. N. Milosavljević.

171.*Trichophaea woolhopeia (Cooke \& W. Phillips) Boud.

Goč Mt. (Cvetne livade), 23.07.2014., N.M. 140723-2, leg. \& det. N. Milosavljević; Fruška Gora Mt. (Erdelj), 09.11.2015., on soil, F.G. 327, leg. \& det. D. Savić; Fruška Gora Mt. (Ravne), 07.07.2016., on soil, leg. \& det. D. Savić; Fruška Gora Mt. (Rakovac vill.), 06.07.2016., on soil, F.G. 476, leg. \& det. D. Savić; Fruška Gora Mt. (Iriški venac), 05.06.2018., on soil, F.G. 861, leg. \& det. D. Savić.

172.*Trichophaeopsis bicuspis (Boud.) Korf \& Erb

Fruška Gora Mt. (Stražilovo), 23.08.2013., on soil, leg. \& det. D. Savić.

173.*Trichophaeopsis paludosa (Boud.) Häffner \& L.G. Krieglst.

Fruška Gora Mt. (Ravne), 11.07.2016., on soil, F.G. 494, leg. \& det. D. Savić.

Fam.: Rhizinaceae Bonord. 1851

\section{4.*Psilopezia nummularia Berk.}

Fruška Gora Mt. (Stražilovo), 25.08.2016., F.G. 472, leg. \& det. D. Savić; Fruška Gora Mt. (Papratski do), 28.08.2016., on Fagus sylvatica, rotten wood, F.G. 473, leg. \& det. D. Savić; Sićevačka klisura, along Nišava r., on a stump of a deciduous tree, 07.10.2018, M.S.N. 257, leg \& det I. Kajevska.

\section{Rhizina undulata Fr.}

Srbija, Karadžić \& Stanivuković 2010: 5, on Pinus nigra; Golija Mt., Karadžić et al. 2017: 26, on Picea abies. 
Fam.: Sarcoscyphaceae Le Gal ex Eckblad 1968

176. Pithya cupressina (Batsch) Fuckel

Deliblatska peščara, Milijašević 2003: 165, on Juniperus virginiana.

177.*Pseudopithyella minuscula (Boud. \& Torrend) Seaver

Deliblatska peščara (Heronje), 10.04.2013, leg. \& det. D. Savić.

178. Sarcoscypha austriaca (O.Beck ex Sacc.) Boud.

Zasavica (Široka bara), Cviyanovicy et al. 2009: 239; Fruška Gora Mt. (Popovica), 15.04.2013., rotten wood, leg. \& det. D. Savić; Kragujevac (Grošnica vill.), 18.04.2014., leg. \& det. N. Milosavljević; Šabac (Mali Zabran), 19.02.2016., on Robinia pseudoacacia, branch, leg. \& det. S. Stanojević.

179. Sarcoscypha coccinea (Jacq.) Sacc.

Beograd (Topčider), Ranojević 1937: 6, on Vitis vinifera, branch; Beograd (Ada Ciganlija), 25.02.1990., leg. \& det. I. Hadžić; Obedska bara (Matijevica-Kadionica), Beronja 1995: 3; Vršačke planine, Matavulj 1995: 60, 61 i 62, on Quercus cerris, Carpinus betulus and Tilia tomentosa; Vršačke planine (Mesić vill. and Sočica vill.), Matavulj et al. 1995: 173, on Quercus cerris; Vršačke planine (Sočica vill. and Markovac vill.), Matavulj et al. 1995: 174, on Carpinus betulus; Vršačke planine (on the northern slopes of Vršački and Gudurički peak), Matavulj et al. 1995: 174, on Tilia tomentosa; Majdanpečka domena-Valley of the Peck r., Karadžić \& Čolić 2009: 30, on Alnus glutinosa and A. incana; Fruška Gora Mt. (Dumbovo, Glavica, Iriški venac, Papratski do, Paragovo, Popovica and Stražilovo), Karaman et al. 2012: 42; Šabac (Gornja Vranjska), 06.03.2015., on Prunus cerasifera, branch, leg. \& det. S. Stanojević; Šabac (Letnjikovac), 24.12. 2017., on Prunus cerasifera, branch, leg. \& det. S. Stanojević; Kukavica Mt. (Slatina vill.), 15.02.2017., on Rubus sp., branch, leg. \& det. S. Jovanović; Goč Mt. (Cvetne livade), 16.04.2014., leg. \& det. N. Milosavljević; Niš (Gabrovac vill.), 24.02.2017., on Tilia cordata, twig, M.S.N. 159, leg. \& det. I. Kajevska.

180. *Sarcoscypha jurana (Boud.) Baral

Fruška Gora Mt. (Letenka), 18.02.2014., Tilia tomentosa, rotten wood, leg. \& det. D. Savić.

Fam.: Sarcosomataceae Kobayasi 1937

181. Plectania melastoma (Sowerby) Fuckel

Kukavica Mt. (Slatina vill.), 25.04.2017., F.G. 787, leg. S. Jovanović, det. D. Savić; Niš (Gabrovac vill.), on deciduous tree, 
twig, M.S.N. 106, Kajevska et al. 2018: 84; Suva planina Mt. (Bojanine vode), on deciduous tree, twig, M.S.N. 107, Kajevska et al. 2018: 84 .

182. Pseudoplectania melaena (Fr.) Sacc.

Tara Mt. (Crveni potok), 07.07.2003., rotten coniferous wood, leg. \& det. B. Uzelac; Goč Mt. (Ribnjak), 07.04.2017., N.M. 170407, leg. \& det. N. Milosavljević.

183. Pseudoplectania nigrella (Pers.) Fuckel

Babušnica (Šanac vill. and Stol vill.), 04.2013., on soil, leg. \& det.

D. Stojanović; Niš (between Gabrovac vill. and Gornje Vlase vill.), on soil, M.S.N. 158, Kajevska et al. 2018: 85; Goč Mt. (Ribnjak), 15.04.2018., N.M. 170415, leg. \& det. N. Milosavljević.

184. * Pseudoplectania sphagnophila (Pers.) Kreisel

Goč Mt. (Ovčarnik), 16.04.2018., N.M. 170404, leg. \& det. N. Milosavljević.

185. Urnula craterium (Schwein.) Fr.

Beograd (Makiš), 13.04.1996., leg. \& det. I. Hadžić; Beograd (Bojčinska šuma), Ivančević \& Davidović 2011: 26; Fruška Gora Mt. (Vorovo), Karaman et al. 2012: 42, deciduous tree, branch; Fruška Gora Mt. (Vizić vill.), 20.03.2018., F.G. 803, leg. J. Gajić, det. D. Savić; Novi Bečej (Miloševački rit), 05.04.2018., leg. D. Ugrnov, det. D. Savić.

186. Urnula mediterranea (M. Carbone, Agnello \& Baglivo) M. Carbone, Agnello \& P. Alvarado

Kragujevac (Žeželj), Milosavljević 2017: 158; Suva planina Mt. (Gadžin han), on Carpinus orientalis, branch, M.S.N. 213, Kajevska et al. 2018: 86.

Fam.: Tuberaceae Dumort. 1822

187. Choiromyces meandriformis Vittad.

Fruška Gora Mt. (Sremska Kamenica), sub Ch. ganglioides, Schulzer et al. 1866: 60; Trstenik (Poljna vill.), Ranojević 1910: 355; Deliblatska peščara, sub Tuber album, Frančišković 1950: 27; Beograd (Bojčinska šuma), 04.08.1992., leg. \& det. I. Hadžić; Babušnica (Linovo vill.), 07.2009., soil, hypogeous, leg. \& det. D. Stojanović; Beograd (Bojčinska šuma), Ivančević \& Davidović 2011: 24; Fruška Gora Mt. (Paragovo), 28.05.2016., leg. B. Šeguljev, det. D. Savić; Fruška Gora Mt. (Vrdnik vill.), 23.06. 2016., F.G. 517, leg. \& det. D. Savić; Beograd (Bojčinska šuma and Lipovička šuma), Boljevac (Rujište), Obedska bara (Debela 
gora), Sopot (Nemenikuće vill.), Tara Mt., Užice (Duboko vill.) and Zlatibor (Kladnica vill.), Ivančević 2016: 71.

188. Choiromyces venosus (Fr.) Th. Fr.

Unknown locality, Ivančević 2016: 72.

189. Paradoxa monospora Mattir.

Tara Mt. (Barski do, Lokvica and Mitrovac-Šljivovica), Ivančević 2016: 97.

190. Tuber aestivum Vittad.

Bela Palanka (Dupeji), Milenković et al. 1997: 226; Bela Palanka and Požarevac, Glamočlija et al. 1997: 213; Babušnica (Resnik vill.), 06.2009., soil, hypogeous, leg. \& det. D. Stojanović; Babušnica (Radinjince vill.), 07.2015., soil, hypogeous, leg. \& det. D. Stojanović; Fruška Gora Mt., Karaman et al. 2012: 42; Zasavica, Savić, D. 25.11.2016., F.G.635, leg. M. Stanković, det. D. Savić; Aleksinac, Belo Polje, Beograd (Bele Vode, Karaula, Košutnjak, Makiš, Zmaj /highway/), Deliblatska peščara (Dumača, Jasenovački mlin, Stražarski mlin), Dobanovci, Fruška Gora Mt., Kosmaj Mt., Lajkovac (Jabučje vill. and Slovac vill.), Malo Crniće (Kalište vill.), Mladenovac (Amerić vill. and Jagnjilo vill. /Mrčevica r./), Obrenovac (Mala Moštanica), Požarevac, Požarevac (Bratinac vill.), Smederevska Palanka, Smederevska Palanka (Glibovac vill.), Suva planina Mt. (Krastavče vill.), Svilajnac (Roanda vill.), Svrljig (Kopajkošara vill.), Šabac (Majur and Veliki Zabran), Tara Mt. (Mitrovac), Užice (Duboko vill.), Valjevo (Gorge of the river Gradac), Velika plana (Veliki lug) and Žitorađa (Dubovo vill.), Ivančević 2016: 106; Fruška Gora Mt. (Stara Bingula), 12.09.2018., F.G. 843, F.G. 855, leg. J. Obradović Milošević, det. D. Savić.

191. Tuber borchii Vittad.

Avala Mt., Sopot (Guberevac vill.-Babe vill.) and Šabac (Veliki Zabran), Ivančević 2016: 107.

192. Tuber brumale Vittadini

Kragujevac-Gornji Milanovac (between Bara vill. and Ljuljak vill.), sub T. melanosporum, Jurišić 1897; Bela Palanka (Dupeji), Svrljig (Crnoljevica vill. and Gramada), sub T. melanosporum, Milenković et al. 1997: 226; Jastrebac, sub T. melanosporum, Lindtner 1935: 15; Pančevo, Frančišković 1950: 27; Beograd (Košutnjak), 01.1997., leg. \& det. I. Hadžić; Svrljig, Bela Palanka and Fruška Gora Mt. (Čortanovci vill.), sub T. melanosporum, Glamočlija et al. 1997: 213; Fruška Gora Mt., Karaman et al. 2012: 43; 
Fruška Gora Mt., sub T. melanosporum, Karaman et al. 2012: 43; Zasavica, Savić, D. 25.11.2016., F.G.633; Beograd (Botanical Garden), Vukojević et al. 2016: 253; Aleksinac (Trnjane vill.), Avala Mt., Banja Koviljača, Barajevo (Arnajevo), Batočina (Rogotska šuma), Bela Palanka (Dupeji), Beograd (Bubanj Potok, Hajdučka česma, Makiš and Zmaj /highway/), Deliblatska peščara (Stražarski mlin), Despotovac (Miliva vill.), Donja Rača, Donja Trnava, Fruška Gora Mt. (Čortanovci vill.), Mladenovac (Jagnjilo vill.), Lajkovac (Mali Borak and Slovac vill.), Lazarevac (Junkovac vill., Sokolovo vill. and Veliki Crljeni vill.), Lipik-Umka, Ljubičevo, Mladenovac (Amerić vill. and Međulužje vill.), Morović (Morović vill.-forest Varadin, Morović vill.-forest Vranjak and "Kućine"), Obrenovac (Veprovica, Zabran and Zabrežje vill.), Pančevo (City forest, Ivanovo vill. and Pančevo-Stari Tamiš), Požarevac, Rača (Saranovo vill.), Rajac Mt., Smederevo (Kolari vill.), Smederevska Palanka, Smederevska Palanka (Glibovac vill.), Sopot (Guberevac vill.-Babe vill., Parcanski vis, Ralja vill. and Zebina voda), Svilajnac (Roanda vill.), Svrljig (Crnoljevica vill. and Gramada), Topola (Maskar vill.), Tresibaba Mt., Trstenik, Ub-Vrelo vill., Užice (Duboko vill.), Valjevo (Bogatić vill., Popare and Popučke) and Velika Plana (Markovac and Veliki lug), Ivančević 2016: 108; Fruška Gora Mt. (Stara Bingula), 12.09.2018., F.G. 888, leg. J. Obradović Milošević, det. D. Savić.

Note: In certain papers, findings of Tuber melanosporum are cited. According to Marjanović et al. (2009) this species is not present in Serbia thus all published data are wrong and should be attributed to the species $T$. brumale.

193.* Tuber dryophilum Tul. \& C. Tul.

Fruška Gora Mt. (Stara Bingula), 05.10.2018., F.G. 813, leg. J. Obradović Milošević, det. D. Savić.

\section{Tuber excavatum Vittad.}

Aleksinac, Batočina, Bela Palanka (Dupeji), Belo Polje, Beograd (Bojčinska šuma, Karaula, Košutnjak and Ostružnica), Deliblatska peščara (Dumača, Jasenovački mlin, Krajnji potok and Stražarski mlin), Fruška Gora Mt., Fruška Gora Mt. (Ljuba vill.), Knjaževac (Balanovac vill.), Kosmaj Mt., Kosmaj Mt. (Košutica), Kupinovo vill., Lajkovac (Jabučje vill. and Slovac vill.), Ljig (Babajić vill.), Mladenovac (Međulužje vill.), Morović (Morović vill.-forest Vranjak and "Kućine"), Obedska bara, Obrenovac (Urovci vill.), Požarevac (Bratinac vill.), Rača (Saranovo vill.), Šabac (Slepčević vill., Štitar vill. and Veliki Zabran), Smederevska Palanka, 
Smederevska Palanka (Glibovac vill.), Sopot (Guberevac vill. Babe vill. and Ralja vill.), Svrljig (Kopajkošara vill., Lozan vill. and Kršina stream), Šabac (Majur), Tara Mt. (Dolak, Mitrovac and Mitrovac - Šljivovica), Užice (Duboko vill.), Valjevo (Bogatić vill. and Gorge of the river Gradac), Velika plana (Veliki lug) and Žitorađa (Dubovo vill.), Ivančević 2016: 110; Babušnica (Vava vill.), 30.10.2017., M.S.N. 210, leg. D. Stojanović, det. I. Kajevska.

195. Tuber ferrugineum Vittad.

Lajkovac (Jabučje vill.), Rača (Saranovo vill.), Tara Mt. (Šljivovica) and Valjevo (Popare), Ivančević 2016: 111.

196. Tuber foetidum Vittad.

Beograd (Bojčinska šuma), 10.10.2004., leg. \& det. I. Hadžić; Beograd (Bojčinska šuma), Ivančević \& Davidović 2011: 26; Bela Palanka (Dupeji) and Beograd (Bojčinska šuma and Makiš), Ivančević 2016: 112.

197. Tuber fulgens Quél.

Tara Mt. (Barski do, Borjak, Dolak, Lokvica, Mitrovac, MitrovacŠljivovica and Tisovo brdo-Lokvica), Ivančević 2016: 113.

198. Tuber macrosporum Vittad.

Obrenovac (Mala Moštanica) and Morović vill., Glamočlija et al. 1993: 96 and Glamočlija et al. 1997: 213; Zasavica, 25.11.2016., F.G.632, leg. M. Stanković, det. D. Savić; Aleksinac, Banatska Palanka, Barajevo (Gradište and Veliki Borak), Batočina (Rogotska šuma), Deliblatska peščara (Jasenovački mlin and Stražarski mlin), Lajkovac (Mali Borak), Lazarevac (Junkovac vill., Sokolovo vill. and Veliki Crljeni vill.), Lipik-Umka, Ljubičevo, Mladenovac (Međulužje vill.), Obrenovac (Zabrežje vill.), Pančevo (City forest), Petrovac na Mlavi (Orljevo vill.), Požarevac, Požarevac (Bratinac vill.), Rača (Saranovo vill.), Rucka (Bukvar), Smederevo (Kolari vill.), Smederevska Palanka, Smederevska Palanka (Glibovac vill.), Sopot (Ralja vill. and Zebina voda), Svilajnac (Roanda vill.), Svrljig (Gramada, Lozan vill. and Svrljig-Požarevac), Trstenik, Uzdinska šuma, Užice (Duboko vill.), Valjevo (Bogatić vill., Gorge of the river Gradac and Popučke) and Velika Plana (Markovac), Ivančević 2016: 114; Fruška Gora Mt. (Stara Bingula), 05.10.2018., F.G. 934, leg. J. Obradović Milošević, det. D. Savić; Morović, 18.10.2018., F.G. 932, leg. J. Obradović Milošević, det. D. Savić. 
199. Tuber maculatum Vittad.

Koviljsko-Petrovaradinski rit, Katanić et. al. 2015: 331; Beograd (Lipovička šuma), Čenta, Deliblatska peščara (Dumača), Lajkovac, Lajkovac (Bajevac vill. and Jabučje vill.), Mionica (Gornji Lajkovac vill.), Obrenovac and Obrenovac (Zabran), Ivančević 2016: 115.

200. Tuber magnatum Picco

Srem and Šumadija, Frančišković 1950: 27; Batočina and Vojvodina, Glamočlija et al. 1997: 213; Zasavica, 20.11.2016., F.G.634, leg. M. Stanković, det. D. Savić; Barajevo (Arnajevo and Gradište), Batočina (Rogotska šuma), Boljevci-Crni Lug, Valley of the Velika Morava r., Lazarevac (Sokolovo vill.), Morović (Morović vill.-forest Varadin), Obrenovac (Zabrežje vill.), Obrež vill., Pančevo (City forest), Petrovac na Mlavi (Rašanac vill.), Rača (Saranovo vill.), Šabac (Slepčević vill.), Smederevo (Kolari vill.), Topola (Natalinci vill.), Ub-Vrelo vill., Crepaja, Valjevo, Valjevo (Bogatić vill.) and Vojvodina, Ivančević 2016: 116.

201. Tuber mesentericum Vittad.

Deliblatska peščara (Dumača), Fruška Gora Mt. (Ljuba vill.), Lazarevac (Sokolovo vill.), Obrenovac (Zabrežje vill.), Šabac (Slepčević vill.), Tara Mt. (Dolak, Šljivovica and Tisovo brdoLokvica), Užice (Duboko vill.) and Valjevo (Popare), Ivančević 2016: 117.

202. Tuber nitidum Vittad.

Beograd (Karaula and Košutnjak), Fruška Gora Mt. (Crveni čot), Rača (Saranovo vill.) and Svrljig (Gramada), Ivančević 2016: 118.

203. Tuber oligospermum (Tul. \& C. Tul.) Trappe

Valjevo (Gorge of the river Gradac and Popare), Ivančević 2016: 119.

204. Tuber petrophilum Milenković, P. Jovan., Grebenc, Ivančević \& Marković

Tara Mt. (Dolak), Milenković et al. 2015: 1143; Tara Mt. (Dolak, Kovači, Mitrovac, Rustine, Tisovo brdo and Tisovo brdo-Lokvica), Ivančević 2016: 119.

205. Tuber puberulum Berk. \& Broome

Bukovica (UTM squares DQ11), Lajkovac (Bajevac vill.), Ravna Gora and Tara Mt. (Tisovo brdo-Lokvica), Ivančević 2016: 120. 
206. Tuber rufum Pollini

Kać, Katanić et al. 2008: 53; Koviljsko-Petrovaradinski rit, Katanić et al. 2015: 331; Banatska Palanka, Banja Koviljača, Bela Palanka (Dupeji), Belo Polje, Beograd (Arboretum of the Faculty of Forestry, Cerak, Hajd park and Karaula), Crepaja, Ćuprija (Mijatovac vill.), Deliblatska peščara (Dumača, Krajnji potok and Stražarski mlin), Dobanovci, Fruška Gora Mt., Fruška Gora Mt. (Crveni Čot and Ljuba vill.), Kupinovo vill., Lajkovac, Lajkovac (Jabučje vill., Mali Borak and Slovac vill.), Lazarevac (Junkovac vill., Sokolovo vill. and Veliki Crljeni vill.), Mladenovac (Jagnjilo vill. /Mrčevica r./), Obrenovac (Zabrežje vill.), Pančevo (City forest and Ivanovo vill.), Požarevac, Rača (Saranovo vill.), Ražanj, Šabac (Štitar vill. and Veliki Zabran), Smederevo (Kolari vill.), Sopot (Babe vill., Guberevac vill.-Babe vill. and Ralja vill.), Suva planina Mt. (Krastavče vill.), Svrljig (Gramada, Guševac vill. and Lozan vill.), Tara Mt. (Barski do, Dolak, Kovači, Lokvica, Mitrovac-Šljivovica, Rustine, Simići, Tisovo brdo, Tisovo brdo-Lokvica, Mitrovac), Ub-Vrelo vill., Umka, Uzdinska šuma, Užice (Duboko vill.), Valjevo (Bogatić vill. and Popučke), Velika plana (Veliki lug) and Žitorađa (Dubovo vill.), Ivančević 2016: 121; Tara Mt. (Dolak-Branevina and Šljivovica), sub Tuber rufum var. apiculatum, Ivančević 2016: 123; Fruška Gora Mt. (Vizić), 06.09.2018., F.G. 865, leg. J. Obradović Milošević, det. D. Savić; Morović, 18.10.2018., F.G. 933, leg. J. Obradović Milošević, det. D. Savić.

\section{CONCLUSIONS}

Report on species distribution from the class Pezizomycetes in Serbia resulted with a total number of 206 taxa distributed in 62 genera and 12 families. Among the species listed 105 species represent new records for the country. The most species dominant families are as follows: Pyronemataceae (78), Pezizaceae (31), Ascobolaceae (22), Helvellaceae (21) and Tuberaceae (19). Although the species list contains data from all parts of the country many regions lack systematic research and the diversity of Pezizomycetes in Serbia remains unknown for the most part. But even though not all regions have been thoroughly investigated, during this study we established remarkable species diversity in the country and therefore we expect more valuable data in the future. So far known summarized data in this paper will significantly contribute to the knowledge of this taxonomic group in the country. 


\section{Acknowledgements}

The authors express sincere gratitude to many colleagues for collecting and sending samples of fungi from various parts of Serbia and for the generous ceding of their unpublished findings (Aleksandar Peškić, Bojan Š eguljev, Branislav Uzelac, Danilo Ugrnov, Draško Lazić, Dejan Stojanović, Draško Grujić, Eleonora Bošković, Gordana Bogićević, Gvozdan Taskov, Ibrahim Hadžić, Ivana Cvijetan, Jelena Biserčić, Jelena Gajić, Jelena Obradović Milošević, Jožef Došai, Marjan Kuštera, Marko Marković, Mile Petrov, Milka Adžić, Monika Slavova, Nebojša Pavković, Nemanja Marić, Nenad Minčić, Radmila Lukić, Selena Jovanović, Sonja Vlahović-Nikolić, Srđan Lazarević, Srđan Stanojević, Stevan Baluban, Slavko Cekić, Vesna Milosavljević, Vojin Lilić, Žarko Jorgovanović).

\section{REFERENCES}

Benkert, D. (2006): Octospora erzbergeri (Pezizales, Ascomycetes), eine neue Art aus Serbien-Montenegro. - Östereichische Zeitschrift für Pilzkunde 15: 1-6.

Benkert, D. (2007): Zur Kenntnis des Vorkommens bryophiler Pezizales (Ascomycota) in Südost-Europa. - Mycologia Montenegrina 10: 7-21.

Cviyanovicy, M., Stankovicy, M., Matavuly, M., Lolicy, S., Pyanicy, B. (2009): Macrofungi of the Zasavica special nature reserve. - Proceedings for Natural Sciences, Matica Srpska, Novi Sad 116: 235-243.

Čolić, D. (1967): Sinekološka analiza flore gljiva u rezervatu sa pančićevom omorikom na Mitrovcu (planina Tara). - Zaštita prirode 34: 389-505.

Eckblad, F-E. (1968): The genera of operculate discomycetes. A re-evaluation of their taxonomy, phylogeny and nomenclature. - Nytt Magasin for Botanik 15: $1-191$.

Frančišković, S. (1950): Naši tartufi. - Šumarski list 74: 23-38.

Glamočlija, J., Vujičić, R., Vukojević, J. (1997): Evidence on Truffles in Serbia. Mycotaxon 65: 211-222.

Hansen, K., Læssøe, T., Pfister, D. H. (2002): Phylogenetic diversity in the core group of Peziza inferred from ITS sequences and morphology. - Mycological Research 106(8): 879-902.

Hansen, K., Perry, B. A., Dranginis, A. W., Pfister, D. H. (2013): A phylogeny of the highly diverse cup-fungus family Pyronemataceae (Pezizomycetes, Ascomycota) clarifies relationships and evolution of selected life history traits. Molecular Phylogenetics and Evolution 67(2): 311-335.

Hansen, K., Pfister, D. H. (2006): Systematics of the Pezizomycetes - the operculate discomycetes. - Mycologia 98: 1029-1040.

Ivančevic, B., Beronja, J. (2004): First records of macromycetes from the Serbian of Stara Planina Mts (Balkan Range). - Mycologia Balcanica 1: 15-19.

Ivančević, B., Davidović, M. (2011): Makromicete na području Bojčinske šume i okvir za njihovo očuvanje. - Zaštita prirode 61(2): 21-33. 
Ivančević, B., Matavulj, M., Vukojević, J., Karaman, M. (2012): Fungi in the legislation of the republic of Serbia. - Zbornik Matice srpske za prirodne nauke 123: 51-64.

Jacobson, A., Kullman, B., Huhtinen, S. (1998): Genus Octospora (Pezizales) in Estonia and Finland. - Karstenia 38: 1-25.

Jurišić, Ž. (1897): Trifa (Tuber). - Delo 13: 138-140.

Kajevska, I., Kuštera, M., Cvijetan, I. (2018): Prilog poznavanju askomiceta istočne Srbije. - Biologica Nyssana 9 (2): 77-88.

Kajevska, I., Savić, D. (In press): First record of Neolecta vitellina (Bres.) Korf \& J.K. Rogers and Sowerbyella fagicola J. Moravec from Serbia. - Biologica Nyssana

Karadžić, D. (2003): Najznačajnije bolesti u bukovim šumama Srbije. - Šumarstvo 1-2: 59-72.

Karadžić, D., Čolić, N. (2009): Najčešće parazitske i saprofitske gljive na stablima crne (Alnus glutinosa Gaertn.) i sive jove (Alnus incana Mnch.). - Glasnik Šmarskog fakulteta Univerziteta u Banjoj Luci 11: 27-36.

Karadžić, D., Milijašević, T. (2004): Najčešće parazitske i saprofitske gljive u izdanačkim šumama bukve u Srbiji. - Šumarstvo 56(3): 25-35.

Karadžić, D., Stanivuković, N. (2010): Najznačajniji fitopatološki problemi u kulturama crnog i belog bora u Srbiji i Republici Srpskoj. - Šumarstvo 3-4: 1-4.

Karaman, M., Novaković, M., Savić, D., Matavulj, M. (2012): Preliminary checklist of Myxomycota and Ascomycotas from Fruška Gora Mountain. Zbornik Matice srpske za prirodne nauke 123: 37-49.

Katanić, M., Grebenc, T., Hrenko, M., Štupar, B., Galić, Z., Orlović, S., Kraigher, H. (2008): Prva identifikacija tipova ektomikorize u zasadu belih topola (Populus alba) kod Novog Sada. - Topola 181/182: 49-59.

Katanić, M., Grebenc, T., Orlović, S., Matavuly, M., Kovačević, B., Bajc, M., Kraigher, H. (2015): Ectomycorrhizal fungal community associated with autochthonous white poplar from Serbia. - iForest 9: 330-336.

Kumar, L. M., Smith, M. E., Nouhra, E. R., Orihara, T., Leiva, P. S., Pfister, D. H., McLaughlin, D. J., Trappe, J. M., Healy, R. A. (2017): A molecular and morphological re-examination of the generic limits of truffles in the TarzettaGeopyxis lineage - Densocarpa, Hydnocystis, and Paurocotylis. - Fungal Biology 121(3): 264-284.

Landvik, S., Egger, K. N., Schumacher, T. (1997): Towards a subordinal classification of the Pezizales (Ascomycota): phylogenetic analyses of SSU rDNA sequences. - Nordic Journal of Botany 17(4): 403-418.

Ławrynowicz, M., Milenković, M., Marković, M., Ivančević, B. (1997): Terfezia terfezioides - a new hypogeous fungus for Balkan Peninsula. - Acta Mycologica 32: 233-238.

Lindtner, V. (1935): Podzemne gljive u Srbiji. - Šumarski list 59: 15-18.

Marjanović, Ž., Grebenc, T., Marković, M., Glišić, A., Milenković, M. (2009): Molecular diversity and ecological specificity of truffles (genus Tuber) originating from mid-west of the Balkan Peninsula. - Sydowia 62(1): 67-87. 
Matavulj, M. (1995): Lignicolous macrofungi of some plant associations of the Vrshachke planine mountains. - Proceedings for Natural Sciences, Matica Srpska, Novi Sad 88: 59-64.

Matavulj, M., Bokorov, M., Stojšić, V. (1995): Prilog proučavanju lignikolnih makromiceta Vršačkih planina. - Zaštita prirode 46-47: 173-176.

Matočec, N., Antonić, O., Mrvoš, D. (1995): The genus Scutellinia (Pezizales, Ascomycotina) in Croatia: preliminary part. - Natura Croatica 4(1): 1-58.

Milenković, M., Glamočlija, J., Veljković, V., Vukojević, J. (1992): Record of two Tuber (T. aestivum Vitt. and T. melanosporum Vitt.) species in Serbia. Archives of biological sciences 44 (3-4): 223-228.

Milenković, M., Grebenc, T., Marković, M., Ivančević, B. (2015): Tuber petrophilum, a new truffle species from Serbia. - Mycotaxon 130: 1141-1152.

Milijašević, T. (2003): Najčešće parazitske i saprofitske gljive na nekim vrstama iz familije Cupressaceae. - Glasnik šumarskog fakulteta Univerziteta $\mathrm{u}$ Beogradu 87: 161-173.

Milosavljević, N. (2015): Prvi nalaz Helvella lactea (Pezizales) u Srbiji. Mycologia Montenegrina 18: 75-87.

Milosavljević, N. (2016): The genus Helvella in central Serbia. - Mycologia Montenegrina 11: 51-96.

Milosavljević, N. (2017): The first record of Urnula mediterranea (Pezizales) in Continental Europe. - Ascomycete org 9(5): 156-163.

Nannfeldt, J. A. (1932): Studien über die morphologie und systematik der nichtlichenisierten inoperculaten discomyceten. - Nova Acta Regiae Societatis Scientiarum Upsaliensis 8: 1-368.

Olariaga, I., Van Vooren, N., Carbone, M., Hansen, K. (2015): A monograph of Otidea (Pyronemataceae, Pezizomycetes). - Persoonia 35: 166-229.

Perić, B., Radić, S. (2013): Deux taxons du genre Morchella (Pezizales), interessants et rares en Europe: M. steppicola et M. eximia. - Mycologia Montenegrina 16: 29-44.

Ranojević, N. (1902): Beitrag zur Pilzflora Serbiens. - Hedwigia 41: 89-103.

Ranojević, N. (1910): Zweiter Beitrag zur Pilzflora Serbiens. - Annales Mycologici 8(3): 347-402.

Ranojević, N. (1914): Dritter Beitrag zur Pilzflora Serbiens. - Annales Mycologici 12(4): 393-421.

Sadiković, D., Kuštera, M. (2013): Fungal conservation: Protected species of fungi in South Serbia region. - Biologica Nyssana 4(1-2): 35-40.

Schulzer, S., Kanitz, A., Knapp, J. A. (1866): Die bisher bekannten Pflanzen Slavoniens, ein Versuch. - Verhandlungen der Zoologisch-Botanischen Gesellschaft Wien 16: 3-172.

Schumacher, T. (1990): The genus Scutellinia (Pyronemataceae). - Opera Botanica 101: 1-107.

Spatafora, J. W., Sung, G-H., Johnson, D., Hesse, C., O’Rourke, B., Serdani, R., Lutzoni, F., Hofstetter, V., Miadlikowska, J., Reeb, V., Gueidan, C., Fraker, 
E., Lumbsch, T., Lucking, R., Schmitt, I., Hosaka, K., Aptroot, A., Roux, C., Miller, A. N., Geiser, D., Hafellner, J., Hestmark, G., Arnold, A. E., Budel, B., Rauhut, A., Hewitt, D., Untereiner, W. A., Cole, M. S., Scheidegger, C., Schultz, M., Sipman, H., Schoch, C. (2006): A five-gene phylogeny of Pezizomycotina. - Mycologia 98: 1018-1028.

Tedersoo, L., Arnold, A. E., Hansen, K. (2013): Novel aspects in the life cycle and biotrophic interactions in Pezizomycetes (Ascomycota, Fungi). - Molecular ecology 22(6): 1488-1493.

Vega, M., Richter, T., Savić, D., Janošík, L. (2018): Octospora pannosa sp. nov. an attractive-spored species on the pleurocarpous moss Brachytheciastrum velutinum. - Herzogia 31(2): $1000-1006$.

Vukojević et al. (2016) (2016): Diversity of macromycetes in the Botanical Garden "Jevremovac" in Belgrade. - Botanica Serbica 40(2): 249-259.

Beug, M., Bessette, A. E., Bessette, A. R. (2014): Ascomycete fungi from North America: a mushroom reference guide. - University of Texas Press.

Boudier, J. L. E. (1907): Histoire et classification des discomyce'tes d'Europe. Paris: Klincksieck.

Breitenbach, J., Kränzlin, F. (1981): Pilze der Schweitz. Ascomyceten (Schlauchpilze). Volume 1. - Verlag Mykologia, Luzern.

Dennis, R. W. G. (ed.) (1981): British Ascomycetes. Revised edition. - J. Cramer, Vaduz, Germany.

Doveri, F. (ed.) (2004): Fungi Fimicoli Italici. - Associazione Micologica Bresadola, Trento, Italy.

Gajić, M. (1984): Flora Goča - Gvozdac. - Šumarski fakultet, Beograd.

Gajić, M. (1989): Flora i vegetacija Golije i Javora. - Šumarski fakultet - Beograd, OOUR Šumarstvo "Golija”, Ivanjica.

Hansen, L., Knudsen, H. (2000): Nordic Macromycetes. 1. Ascomycetes. Nordsvamp, Kopenhagen.

Ivančević, B. (2016): Prostorna distribucija i ekološke varijacije staništa hipogeičnih macromiceta (Mycota) u Srbiji. - Univerzitet u Beogradu, Biološki fakultet. (PhD Thesis) [in Serbian]

Karadžić, D., Milanović, S., Golubović Ćurguz, V. (2017): Uzroci sušenja smrče (Picea abies Karst.) na području Parka prirode „Golija“. - Univerzitet u Beogradu, Šumarski fakultet, Beograd.

Kirk, P. M., Cannon, P. F., Minter, D. W., Stalpers, J. A. (2008): Dictionary of the Fungi, ed. 10. - CABI, Wallingford, UK.

Montecchi, A., Sarasini, M. (2000): Funghi ipogei D`Europa. - A.M.B. Fondazione, Centro Studi Micologici.

Ranković, B. (1955): Prilog poznavanju gljiva naših rezervata - (gljive Fruške gore). - Zavod za zaštitu prirode i naučno proučavanje prirodnih retkosti Narodne Republike Srbije. Naučna knjiga, Beograd. 
Ranojević, N. (1937): Četvrti prilog poznavanju gljiva u Srbiji. - Glasnik srpske kraljevske akademije 177, Prvi razred 87: 207-223.

Savić, D. (2016): Diverzitet gljiva razdela Ascomycota na području Fruške gore sa posebnim osvrtom na red Helotiales. - Prirodno-matematički fakultet, Univerzitet u Novom Sadu. (PhD Thesis) [in Serbian]

Beronja, J. (1995): Prilog poznavanju makromiceta šireg područja Obedske bare. In: Puzović, S. (ed.): Povratak Obedskoj bari 1: 1-9 [Edicija povratak ibisa]. Mladi istraživači Srbije, Beograd.

Glamočlija, J., Milenković, M, Vujičić, R. (1993): Prvi nalazi Tuber macrosporum Vitt. u Srbiji. In: X Simpozijum jugoslovenskog društva za fiziologiju biljaka, Zemun-Polje, Beograd, 17-18.06.1993.: 96. - Beograd.

Pfister, D. H. (2015): Chapter 2. Pezizomycotina: Pezizomycetes, Orbiliomycetes. The Mycota VII (B). In: Mclaughlin, D. J., Spatafora, J. W. (eds): Systematics and Evolution: 35-55. - Springer-Verlag.

Kirk, P. M., Ansell, A. E. (2004): Authors of Fungal Names. Electronic edition. CABI, Wallingford. [http://www.indexfungorum.org/FungalNameAuthors.doc] (accessed 24.12.2018)

\section{ЧЕК ЛИСТА ГЉИВА КЛАСЕ РЕZIZOMYCETES У СРБИЈИ}

\section{Р Е 3 И М Е}

У овом раду представљен је приказ распрострањености врста из класе Pezizomycetes на територији Републике Србије. Списак врста садржи све досадашње објављење и необјављене податке о налазима. До сада је регистровано укупно 206 врста, од којих су чак 105 нове за земљу. Овај свеобухватни списак врста значајно обогаћује познавање ове таксономске групе у нашој земљи. 